\title{
Good Disclosure, Bad Disclosure
}

\author{
Itay Goldstein and Liyan Yang*
}

January, 2014

\begin{abstract}
We study the real-efficiency implications of public information in a model where relevant decision makers learn from the financial market to guide their actions. Whether disclosure is "good" or "bad" depends on the interactions between two effects on real decision makers' forecast. Disclosure has a positive direct effect of providing new information, and it also has an indirect effect of changing the price informativeness. If disclosure is about a variable of which real decision makers are well informed, then the indirect effect is also positive, and overall disclosure improves real efficiency. If disclosure is about a variable that real decision markers care to learn much, then the indirect effect is negative, and it dominates the positive direct effect if and only if the market aggregates information effectively.
\end{abstract}

Keywords: Disclosure, Price Informativeness, Feedback Effects, Real Efficiency.

JEL Classifications: D61, G14, G30, M41

*Itay Goldstein: Department of Finance, Wharton School, University of Pennsylvania, Philadelphia, PA 19104; Email: itayg@wharton.upenn.edu; Tel: 215-746-0499. Liyan Yang: Department of Finance, Joseph L. Rotman School of Management, University of Toronto, 105 St. George Street, Toronto, Ontario M5S 3E6; Email: liyan.yang@rotman.utoronto.ca; Tel: 416-978-3930. We would like to thank the seminar participants of Theory Lunch Workshop and Rotman Brownbag Workshop at University of Toronto. 


\section{Introduction}

There is abundant public information in reality. Government agencies, such as the Bureau of Economic Analysis, frequently publish economic statistics about the economic conditions. Corporate firms, either mandatorily or voluntarily, make various announcements about their operations to the public. In effect, regulators and academics often view disclosure as one of the most powerful policy tools, ${ }^{1}$ and many recent government policies, such as SarbanexOxley, RegFD, and Dodd-Frank, are involved with increased disclosure requirements. ${ }^{2}$ One underlying premise for promoting public disclosure is that it can improve real efficiency by providing new information to relevant decision makers. For example, the FASB states: "The benefits of financial reporting information include better investment, credit, and similar resource allocation decisions, which in turn result in more efficient functioning of the capital markets and lower costs of capital for the economy as a whole." ${ }^{3}$ In this paper, we propose a framework to examine whether and when this premise holds.

In our model economy, speculators trade one risky asset in the financial market based on their private and public information. The cash flow of the risky asset is in turn determined by the relevant decision makers who have access to a production technology. Real decision makers use the pubic information and more importantly, the asset price formed in the financial market, to guide their investments. ${ }^{4}$ It is their actions that establish the effect that public information has on the real economy. Our mechanism works through the interactions between the exogenous public information and the endogenous price information, both of which affect the forecast quality of real decision makers.

The production technology determining the asset cash flow is involved with two indepen-

\footnotetext{
${ }^{1}$ Greenstone et al. (2006, p. 399) state: "Since the passage of the Securities Act of 1933 and the Securities Exchange Act of 1934, the federal government has actively regulated U. S. equity markets. The centerpiece of these efforts is the mandated disclosure of financial information."

${ }^{2}$ In effect, Sarbanes-Oxley Act was passed as an "act to protect investors by improving the accuracy and reliability of corporate disclosures made pursuant to the securities laws, and for other purposes."

${ }^{3}$ FASB Financial Accounting Series, NO.1260-001 July 6 2006, "Conceptual Framework for Financial Reporting: Objective of Financial Reporting and Qualitative Characteristics of Decision-Useful Financial Reporting Information," Section QC53, p. 35.

${ }^{4}$ Recent empirical studies document that asset prices indeed contain valuable information relevant to real decisions and that decision makers also appear to learn information from prices (e.g., Luo, 2005; Chen, Goldstein, and Jiang, 2007; Edmans, Goldstein, and Jiang, 2012; Foucault and Frésard, 2013). See Bond, Edmans, and Goldstein (2012) for a recent survey.
} 
dent productivity factors - factor $\tilde{a}$ and factor $\tilde{f}$ - such as a macro factor and a firm-specific factor as in Veldkamp and Wolfers (2007), or a permanent factor and a transitory factor as in Liu, Wang, and Zha (2013). Real decision makers know better about one factor $(\tilde{a})$ than the other $(\tilde{f})$, and hence they are more keen to learn about the factor $(\tilde{f})$ of which they are relatively uninformed. Speculators have private information about both productivity factors $\tilde{a}$ and $\tilde{f}$. The equilibrium asset price will therefore convey information about $\tilde{a}$ and $\tilde{f}$ through their trading, which is useful for real decision makers to make investments.

The key insight emerging from our analysis is that whether public disclosure is "good" in terms of promoting real efficiency depends on whether it makes speculators trade on the right private information such that the overall forecast quality of real decision makers gets improved. In general, public disclosure has two effects on real decision makers' forecast quality. The direct effect is to provide new information, and it is always positive, as real decision makers always become better informed after observing some information, however noisy. The second effect is an indirect effect: Public disclosure affects speculators' trading, which in turn affects the price informativeness about factor $\tilde{f}$ that real decision makers care to learn. This indirect effect can be positive or negative, and when it is negative, it can even dominate the direct positive effect.

When the public disclosure is primarily a signal about factor $\tilde{a}$ of which real decision makers have already had a good knowledge, both the direct and indirect effects are positive. To see this, note that the speculators' trading is determined by the public signal about $\tilde{a}$ and their private signals about $\tilde{a}$ and $\tilde{f}$, since the future asset cash flows are affected by both factors $\tilde{a}$ and $\tilde{f}$ and these signals convey information about these factors. When the public signal mainly provides information about $\tilde{a}$ and when it becomes more accurate, speculators will put more weight on this public information and less on their private information in forecasting factor $\tilde{a}$. As a result, their private-information-based trading will reflect more of their private information about factor $\tilde{f}$ that real decision makers care to learn. That is, the indirect effect of the price informativeness is also to benefit real decision makers' learning. So, disclosing information about factor $\tilde{a}$ will improve the forecast quality of real decision makers and hence real efficiency.

When the public disclosure is mainly a signal about factor $\tilde{f}$ that real decision makers 
wish to learn, the indirect effect becomes negative. This is because a more accurate public signal about $\tilde{f}$ will render speculators to put less weight on their own private information about $\tilde{f}$, which reduces the informational content about $\tilde{f}$ in the price. This negative effect of disclosure is strong when the market effectively aggregates speculators' private information, so that the overall effect of disclosure is to harm real decision makers' learning quality and harm real efficiency. If the market does not aggregate private information effectively, then the positive direct effect of disclosure still dominates, and so disclosing information about $\tilde{f}$ still improves real efficiency.

The punchline of our analysis can therefore be summarized as follows. Market prices convey information to relevant decision makers, and the fluctuation of market prices is driven by multiple factors. Disclosing public information is "good" only when it improves the overall learning quality of relevant real decision makers. This is more likely to occur when the information being disclosed is about something that real decision makers have already known much, or when the market does not aggregate traders' private information effectively if the disclosure is about something that real decision makers wish to learn. More generally, our model provides a flexible framework to study financial trading and price informativeness in the presence of feedback effects and multiple sources of uncertainty, and such a framework can be used to analyze links among public and private information, asset prices, and real efficiency.

As an application of our analysis, consider the following concrete example (that we also use in developing our model). The asset is the stock on a financially-constrained firm, who needs fund from capital providers (such as banks) to make investments. The real decision makers in this example are the capital providers. Factor $\tilde{a}$ can be information related to the aggregate industry, while factor $\tilde{f}$ is the firm-specific information. It seems reasonable that capital providers might have better information about the whole industry than the specific firm. If the disclosure is coming from the government, it is more likely to be about the aggregate economy, and according to our theory, this kind of disclosure is good in promoting real efficiency. However, if the disclosure is coming from the firm, such disclosure should be more cautious. This is because this kind of information is more likely firm specific, and whether such a disclosure is good or bad depends on whether the market price has already 
aggregated much information about the firm.

\section{Related Literature}

A a broad level, our paper complements two literatures: the accounting literature on the real effects of accounting information disclosure, and the finance literature on the real effects of financial markets (which is labeled "feedback effects" in this literature). Kanodia (2007) and Bond, Edmans, and Goldstein (2012) provide a review of these two literatures, respectively. Our paper introduces the interactions among public disclosure, trading by informed speculators, and learning by real decision makers, and provides new real-efficiency implications of disclosure based on these interactions. Below, we discuss in detail several studies that also explore adverse efficiency implications of public information through various mechanisms and are thus most closely related to our paper.

Amador and Weill (2010) construct a monetary model and show that releasing public information about monetary and/or productivity shocks can reduce welfare through reducing the informational efficiency of the price system, which is similar to the efficiency-reducing channel in our financial model. Our analysis is different from Amador and Weill (2010) in two important ways. First, in their macro framework, workers learn from good prices, while in our trading framework, real decision makers are company managers and capital providers, who learn from financial asset prices. As Dow and Gorton (1997) argue, financial markets and good markets are quite different in many dimensions, which is particularly true in the aspect of information transmission. Second, the results are different. In Amador and Weill (2010), the indirect effect of pubic disclosure about any type of information (monetary shocks or productivity shocks) on workers' forecast quality is always negative. By contrast, our analysis emphasizes that the indirect effect of public disclosure can be either positive or negative, depending on what type of information disclosure conveys.

Bond and Goldstein (2012) analyze a trading model where a government learns from asset prices and intervenes in the asset cash flows. Their analysis also suggests that disclosure can either reduce or raise price informativeness, depending on the type of information being disclosed. However, both their mechanism and results are different from ours. First, 
their mechanism works through a risk-return trade off faced by risk-averse traders. On the one hand, disclosure affects the importance of traders' own information in predicting the government's private information that affects asset cash flows; on the other hand, it affects the uncertainty faced by traders through influencing their information sets. In our model, all agents are risk neutral and so our results are not driven by any kind of risk-return tradeoff. Second, in Bond and Goldstein (2012), disclosing information about a variable that the government wishes to learn - i.e., the counterpart of public information about $\tilde{f}$ in our model - makes the price completely useless and its overall effect is to harm the government's intervention decision. By contrast, in our model, although such public disclosure can be detrimental to price informativeness, the positive direct effect of disclosure providing new information may still dominate. So, the overall effect of disclosing information that real decision makers wish to learn can be positive or negative, depending on the effectiveness of information-aggregation in financial markets.

Edmans, Heinle, and Huang (2013), Gao and Liang (2013), and Han et al. (2013) study optimal disclosure by trading off the benefits and costs of disclosure. In all three papers, disclosure harms investment efficiency, albeit through different channels. In Edmans, Heinle, and Huang (2013), only hard information (such as earnings) can be disclosed, and disclosing hard information distorts manager's investment incentives by changing the relative weight between hard and soft information. In Han et al. (2013), disclosure attracts noise trading which reduces price informativeness and harms managers' learning quality. In Gao and Liang (2013), disclosure crowds out private information production, reduces price informativeness, and so harms managers' learning and investments. Here, we study whether and when disclosure harms or improves real decisions, and our results are not driven by anything related to incentives, noise trading attraction, or private-information production.

Some early papers, such as Hirshleifer (1971) and Hakannson, Kunkel, and Ohlson (1982), have pointed out that public information destroys risk-sharing opportunities and thereby impairs the social welfare. In our paper, all agents are risk neutral, and our focus is on real investment efficiency instead of risk sharing. Gao (2010) and Cheynel (2013) have recently considered the welfare implications of corporate disclosure, but both papers do not allow real decision makers to learn from the asset price, which is the key in driving 
our results. A recent line of research rely on payoff externality and hence coordination motives across economic agents to show that public information release may harm welfare, for example, Morris and Shin (2002), Angeletos and Pavan (2004), Angeletos and Pavan (2007), and Goldstein, Ozdenoren and Yuan (2011). In contrast with this line of work, our results are not driven by any kind of payoff externality - speculators do not care about what other speculators do in our setting. Instead, our results are generated from the indirect effect of disclosure on real decision makers' forecast quality through changing the price informativeness.

\section{The Model}

\subsection{Environment}

We consider a variation of the models studied by Goldstein, Ozdenoren, and Yuan (2013) and Goldstein and Yang (2013). There are three dates, $t=0,1,2$. At date 0 , a continuum $[0,1]$ of "speculators" trade one risky asset based on their diverse private signals and a common public signal about factors related to the asset's future cash flows, and the equilibrium asset price aggregates their information through their trading. At date 1, a continuum [0,1] of "real decision makers," who see the public signal and the equilibrium asset price, make inference from the price to guide their actions, which in turn determine the cash flow of the risky asset that was traded in the previous period. At date 2, the cash flow is realized, and all agents get paid and consume.

Our model admits two interpretations, one micro and one macro. At the micro level, the risky asset can be interpreted as a stock of a financially-constrained firm which needs capital from outside capital providers to make investments. The real decision makers in this case are the capital providers, such as banks, equity investors, and venture capital firms. At the macro level, the risky asset can be interpreted as an index on a particular industry or on the aggregate stock market, and the real decision makers are the managers of those companies included in the index. In both interpretations, speculators can be thought of as mutual and/or hedge funds who have private information about the future value of the asset. 
We are agnostic to the two interpretations, but for simplicity, we have adopted the first one and call real decision makers as capital providers in presenting the model.

\subsection{Investment}

The firm in our economy has access to the following production technology:

$$
q\left(k_{j}\right)=\tilde{A} \tilde{F} k_{j}
$$

where $k_{j}$ is the amount of capital that the firm raises from capital provider $j$ at date $1, q\left(k_{j}\right)$ is the date- 2 output that is generated by the investment $k_{j}$, and $\tilde{A} \geq 0$ and $\tilde{F} \geq 0$ are two productivity factors. Let $\tilde{a}$ and $\tilde{f}$ denote the natural $\operatorname{logs}$ of $\tilde{A}$ and $\tilde{F}$, i.e., $\tilde{a} \equiv \log \tilde{A}$ and $\tilde{f} \equiv \log \tilde{F}$. We assume that $\tilde{a}$ and $\tilde{f}$ are normally distributed:

$$
\tilde{a} \sim N\left(0, \frac{1}{\tau_{a}}\right) \text { and } \tilde{f} \sim N\left(0, \frac{1}{\tau_{f}}\right),
$$

where $\tau_{a}>0, \tau_{f}>0$, and $\tilde{a}$ and $\tilde{f}$ are mutually independent. ${ }^{5}$

Factors $\tilde{A}$ and $\tilde{F}$ represent two dimensions of uncertainty that affect the cash flow of the traded firm. For example, one dimension can be a factor related to aggregate economy, and the other one can be firm-specific (e.g., Greenwood, MacDonald, and Zhang, 1996, p. 97; Veldkamp and Wolfers, 2007). Also, $\tilde{A}$ can be thought of as the permanent component in the total productivity and the factor $\tilde{f}$ is the transitory component, as in Liu, Wang, and Zha (2013, p. 1154-1155). Several papers in the finance literature have also specified that the value of the traded security is affected by more than one fundamental; e.g., Froot, Scharfstein, and Stein (1992), Goldman (2005), and Kondor (2012), among others. In Section 4.1.1, we will show that this feature of two-dimensional uncertainty is important for generating our results.

We assume that capital providers have better information about one factor than the other. In the baseline model analyzed in this section, we consider an extreme version of this asymmetric knowledge - i.e., capital providers know perfectly factor $\tilde{A}$ but nothing about factor $\tilde{F}$ beyond the prior distribution. The capital providers are essentially identical in the baseline model - i.e., they have access to the same investment technology and information

\footnotetext{
${ }^{5}$ The assumption that $\tilde{a}$ and $\tilde{f}$ have a mean of 0 is without loss of generality. Assuming non-zero means is equivalent to renormalizing the cost parameter $c_{0}$ introduced shortly.
} 
set. In Section 6, we will extend our model to equip each capital provider with differential noisy signals about the two factors, and show that our results go through as long as the signal quality about one factor is sufficiently different from the signal quality about the other.

At date $t=1$, each capital provider $j$ chooses the level of capital (and investment) $k_{j}$. As in in Goldstein, Ozdenoren, and Yuan (2013), providing capital incurs a private cost according to the following functional form:

$$
c\left(k_{j}\right)=\frac{1}{2} c k_{j}^{2}
$$

where the constant $c>0$ controls the size of the cost relative to the output $q\left(k_{j}\right)$. The cost can be the monetary cost of raising the capital or the effort incurred in monitoring the investment.

We also follow Goldstein, Ozdenoren, and Yuan (2013) and assume that each capital provider $j$ captures proportion $\beta \in(0,1)$ of the full output $q\left(k_{j}\right)$ by providing $k_{j}$, and thus his payoff from the investment is $\beta q\left(k_{j}\right)$. Capital provider $j$ chooses $k_{j}$ to maximize the payoff $\beta q\left(k_{j}\right)$ he captures from the firm minus his cost $c\left(k_{j}\right)$ of raising capital, conditional on his information set. All capital providers have the same information set, denoted by $\mathcal{I}_{R}$ (with the subscript " $R$ " indicating "real decision makers"), which consists of factor $\tilde{A}$, a public signal $\tilde{\omega}$, and the asset price $\tilde{P}$ (we will elaborate on $\tilde{\omega}$ and $\tilde{P}$ in the subsequent subsections shortly). Therefore, capital provider $j$ chooses $k_{j}$ to solve

$$
\max _{k_{j}} E\left[\beta \tilde{A} \tilde{F} k_{j}-\frac{1}{2} c k_{j}^{2} \mid \mathcal{I}_{R}\right] .
$$

The solution to this maximization problem is:

$$
k_{j}^{*}=\frac{\beta \tilde{A} E\left(\tilde{F} \mid \mathcal{I}_{R}\right)}{c} .
$$

\subsection{Public and Private Information}

All agents, including speculators and capital providers, observe a public signal $\tilde{\omega}$, which communicates a linear combination of the two productivity factors with some error; that is,

$$
\tilde{\omega}=\mu_{a} \tilde{a}+\mu_{f} \tilde{f}+\tilde{\varepsilon}_{\omega}
$$

where $\mu_{a}$ and $\mu_{f}$ are two constants, and $\tilde{\varepsilon}_{\omega} \sim N\left(0, \frac{1}{\tau_{\omega}}\right)$ (with $\tau_{\omega}>0$ ) is independent of $\{\tilde{a}, \tilde{f}\}$. The constants $\mu_{a}$ and $\mu_{f}$ determine what information the signal $\tilde{\omega}$ conveys. If 
$\frac{\operatorname{Var}\left(\mu_{a} \tilde{a}\right)}{\operatorname{Var}\left(\mu_{f} \tilde{f}\right)}$ is large, then the variations in $\mu_{a} \tilde{a}+\mu_{f} \tilde{f}$ are largely driven by factor $\tilde{a}$, and so $\tilde{\omega}$ is mainly a signal about $\tilde{a}$. If $\frac{\operatorname{Var}\left(\mu_{a} \tilde{a}\right)}{\operatorname{Var}\left(\mu_{f} \tilde{f}\right)}$ is small, then by the same reason, $\tilde{\omega}$ is primarily a signal about factor $\tilde{f}$.

The parameter $\tau_{\omega}$ controls the precision of the public signal $\tilde{\omega}$. The public signal can represent public announcements made by a firm, and the firm can influence the disclosure precision by, for example, affecting the degree of access they may provide to outside analysts. Alternatively, the public signal can be some economics statistics published by government agencies. For example, a current policy debate is on how much information governments should release about the outcomes of bank stress tests, and this amount of public information corresponds to the size of $\tau_{\omega}$ in our model. In our analysis, we will follow the literature (e.g., Morris and Shin, 2002; Amador and Weill, 2010) and conduct comparative statics exercises with respect to the parameter $\tau_{\omega}$ to examine the real-efficiency effect of public information.

In addition to the public signal $\tilde{\omega}$, each speculator $i$ observes two noisy signals about $\tilde{a}$ and $\tilde{f}$, respectively:

$$
\tilde{x}_{i}=\tilde{a}+\tilde{\varepsilon}_{x, i} \text { and } \tilde{y}_{i}=\tilde{f}+\tilde{\varepsilon}_{y, i},
$$

where $\tilde{\varepsilon}_{x, i} \sim N\left(0, \frac{1}{\tau_{x}}\right)$ (with $\left.\tau_{x}>0\right), \tilde{\varepsilon}_{y, i} \sim N\left(0, \frac{1}{\tau_{y}}\right)$ (with $\tau_{y}>0$ ), and they are mutually independent of $\{\tilde{a}, \tilde{f}\}$. Therefore, speculator $i$ 's information set is $\mathcal{I}_{i}=\left\{\tilde{x}_{i}, \tilde{y}_{i}, \tilde{\omega}\right\}$. The market price $\tilde{P}$ will aggregate their private signals $\left\{\tilde{x}_{i}, \tilde{y}_{i}\right\}$ through their trading in the financial market, and hence $\tilde{P}$ will contain information about $\tilde{a}$ and $\tilde{f}$, which is useful for capital providers to make real investment decisions. We next elaborate on the formation of prices.

\subsection{Trading and Price Formation}

At date $t=0$, speculators submit market orders as in Kyle (1985) to trade the risky asset in the financial market. They can buy or sell up to a unit of the risky asset, and thus speculator $i$ 's demand for the asset is $d(i) \in[-1,1]$. This position limit can be justified by borrowing/short-sales constraints faced by speculators. As argued by Goldstein, Ozdenoren, and Yuan (2013), the specific size of this position limit is not crucial, and what is crucial is that speculators cannot take unlimited positions. Speculators are risk neutral, and therefore 
they will choose their positions to maximize the expected trading profits conditional on their information sets $\mathcal{I}_{i}=\left\{\tilde{x}_{i}, \tilde{y}_{i}, \tilde{\omega}\right\}$.

The traded asset is a claim on the portion of the aggregate output that remains after removing capital providers' share. ${ }^{6}$ Specifically, the aggregate output is

$$
\tilde{Q} \equiv \int_{0}^{1} q\left(k_{j}\right) d j=\tilde{A} \tilde{F} \int_{0}^{1} k_{j} d j=\tilde{A} \tilde{F} K .
$$

So, after removing the $\beta$ fraction of $\tilde{Q}$, the remaining $(1-\beta)$ fraction constitutes the cash flow on the risky asset:

$$
\tilde{V}=(1-\beta) \tilde{Q}=(1-\beta) \tilde{A} \tilde{F} K
$$

A speculator's profit from buying one unit of the asset is given by $\tilde{V}-\tilde{P}$, and similarly, his profit from shorting one unit is $\tilde{P}-\tilde{V}$. So, speculator $i$ chooses demand $d(i)$ to solve:

$$
\max _{d(i) \in[-1,1]} d(i) E\left[(1-\beta) \tilde{A} \tilde{F} K-\tilde{P} \mid \mathcal{I}_{i}\right] .
$$

Since each speculator is atomistic and is risk neutral, he will optimally choose to either buy up to the one-unit position limit, or short up to the one-unit position limit. We denote the aggregate demand from speculators as $D \equiv \int_{0}^{1} d(i) d i$, which is the fraction of speculators who buy the asset minus the fraction of those who short the asset.

As in Goldstein, Ozdenoren, and Yuan (2013), to prevent a price that fully reveals the factor $\tilde{f}$ to capital providers, we assume the following noisy supply curve provided by (unmodeled) liquidity traders:

$$
L(\tilde{\xi}, \tilde{P}) \equiv 1-2 \Phi(\tilde{\xi}-\lambda \log \tilde{P})
$$

where $\tilde{\xi} \sim N\left(0, \frac{1}{\tau_{\xi}}\right)$ (with $\tau_{\xi}>0$ ) is an exogenous demand shock independent of other shocks in the economy. As usual, the noisy supply represent trading coming from (unmodeled) agents who trade for liquidity or hedging needs (e.g., Dow and Rahi, 2003). We do not endogenize the actions of these traders in our setting, because doing so here breaks the loglinear structure of the model, which makes impossible an analytical characterization. So, our analysis has focused on real investment efficiency without going further to a full welfare analysis.

In (4), function $\Phi(\cdot)$ denotes the cumulative standard normal distribution function, which

\footnotetext{
${ }^{6}$ As explained in Goldstein, Ozdenoren, and Yuan (2013), for technical reasons, we do not assume that the asset is a claim on the net return from the investment.
} 
is increasing. Thus, the supply curve $L(\tilde{\xi}, \tilde{P})$ is strictly increasing in the price $\tilde{P}$ and decreasing in the demand shock $\tilde{\xi}$. The parameter $\lambda$ captures the elasticity of the supply curve with respect to the price, and it can be interpreted as the liquidity of the market in the sense of price impact: When $\lambda$ is high, the supply is very elastic with respect to the price and thus, the demand from informed speculators can be easily absorbed by noise trading without moving the price very much.

The market clears by equating the aggregate demand $D$ from speculators with the noisy supply $L(\tilde{\xi}, \tilde{P})$ :

$$
D=L(\tilde{\xi}, \tilde{P}) .
$$

This market clearing condition will determine the equilibrium price $\tilde{P}$.

\subsection{Equilibrium Definition}

The exogenous parameters in our model are: $\tau_{a}$, the prior precision of the productivity factor $\tilde{a} ; \tau_{f}$, the prior precision of the productivity factor $\tilde{f} ; \tau_{\omega}$, the precision of the public information; $\tau_{x}$, the precision of speculators' private signals about factor $\tilde{a} ; \tau_{y}$, the precision of speculators' private signals about factor $\tilde{f} ; \tau_{\xi}$, the precision of noise trading; $\lambda$, the elasticity of noisy supply; $\mu_{a}$, the loading on factor $\tilde{a}$ in the public signal; $\mu_{f}$, the loading on factor $\tilde{f}$ in the public signal; $\beta$, the fraction of the output captured by capital providers; and $c$, the parameter controlling the relative size of costs. So, the tuple

$$
\mathcal{E}=\left\{\tau_{a}, \tau_{f}, \tau_{\omega}, \tau_{x}, \tau_{y}, \tau_{\xi}, \lambda, \mu_{a}, \mu_{f}, \beta, c\right\}
$$

defines an economy. For a given economy, we consider rational-expectations equilibrium (REE) which involves the optimal decisions of each player (capital providers and speculators) and the statistical behavior of aggregate variables $(K, D$, and $\tilde{P})$.

Each player's optimal decisions will be a function of their information sets. For capital providers, their optimal investments $k_{j}^{*}$ given by (1) will be a function of their information set $\mathcal{I}_{R}=\{\tilde{a}, \tilde{P}, \tilde{\omega}\}$; that is, $k_{j}^{*}=k(\tilde{a}, \tilde{P}, \tilde{\omega})$. Since they are identical, the aggregate investment function $K(\tilde{a}, \tilde{P}, \tilde{\omega})$ will be the same as the individual investment function: $K=K(\tilde{a}, \tilde{P}, \tilde{\omega})=k(\tilde{a}, \tilde{P}, \tilde{\omega})$. Speculators' optimal trading strategies $d_{i}^{*}$ will be a function of their information set $\mathcal{I}_{i}=\left\{\tilde{x}_{i}, \tilde{y}_{i}, \tilde{\omega}\right\}$; that is, $d_{i}^{*}=d\left(\tilde{x}_{i}, \tilde{y}_{i}, \tilde{\omega}\right)$. In aggregate, the noise 
terms $\tilde{\varepsilon}_{x, i}$ and $\tilde{\varepsilon}_{y, i}$ in their signals $\tilde{x}_{i}$ and $\tilde{y}_{i}$ will wash out, and so the aggregate demand $D$ for the risky asset is a function of $\tilde{a}, \tilde{f}$ and $\tilde{\omega}$ :

$$
D=D(\tilde{a}, \tilde{f}, \tilde{\omega})=\int_{0}^{1} d\left(\tilde{x}_{i}, \tilde{y}_{i}, \tilde{\omega}\right) d i=E\left[d\left(\tilde{x}_{i}, \tilde{y}_{i}, \tilde{\omega}\right) \mid \tilde{a}, \tilde{f}, \tilde{\omega}\right],
$$

where the expectation is taken over $\left(\tilde{\varepsilon}_{x}, \tilde{\varepsilon}_{y}\right)$ in $(6)$.

The market clearing condition (5) will therefore determine the price $\tilde{P}$ as a function of productivity factors $\{\tilde{a}, \tilde{f}\}$, the public signal $\tilde{\omega}$, and the noise trading shock $\tilde{\xi}$ : $\tilde{P}=$ $P(\tilde{a}, \tilde{f}, \tilde{\omega}, \tilde{\xi})$. An REE is defined formally as follows.

Definition 1 A rational-expectations equilibrium consists of a price function, $P(\tilde{a}, \tilde{f}, \tilde{\omega}, \tilde{\xi})$ : $\mathbb{R}^{4} \rightarrow \mathbb{R}$, an investment policy for capital providers, $k(\tilde{a}, \tilde{P}, \tilde{\omega}): \mathbb{R}^{3} \rightarrow \mathbb{R}$, a trading strategy of speculators, $d\left(\tilde{x}_{i}, \tilde{y}_{i}, \tilde{\omega}\right): \mathbb{R}^{3} \rightarrow[-1,1]$, and the corresponding aggregate aggregate demand function for the asset $D(\tilde{a}, \tilde{f}, \tilde{\omega})$, such that:

(a) For capital provider $j, k(\tilde{a}, \tilde{P}, \tilde{\omega})=\frac{\beta \tilde{A} E(\tilde{F} \mid \tilde{a}, \tilde{P}, \tilde{\omega})}{c}$;

(b) For speculator $i, d\left(\tilde{x}_{i}, \tilde{y}_{i}, \tilde{\omega}\right)$ solves (3);

(c) The market clearing condition (5) is satisfied; and

(d) The aggregate asset demand is given by (6).

\section{Equilibrium Characterization}

In this section, we illustrate the steps for constructing an equilibrium. It turns out that the equilibrium characterization boils down to a fixed-point problem of solving the weight that speculators put on the signal $\tilde{y}_{i}$ about the factor $\tilde{f}$ when they trade the risky asset. Specifically, we first conjecture a trading strategy of speculators and use the market clearing condition to determine the asset price and hence the information that capital providers can learn from the price. We then update capital providers' beliefs and characterize their investment rule, which in turn determines the cash flow of the traded asset. Finally, given the implied price and cash flow in the first two steps, we solve for speculators' optimal trading strategy, which compares with the initial conjectured trading strategy to complete the fixed-point loop. 


\subsection{The Information that Capital Providers Learn from the Price}

We conjecture that speculators buy the asset if and only if a linear combination of their (private and public) signals is above a cutoff $g$, and sell it otherwise. That is, speculators buy the asset whenever $\tilde{x}_{i}+\phi_{y} \tilde{y}_{i}+\phi_{\omega} \tilde{\omega}>g$, where $\phi_{y}, \phi_{\omega}$, and $g$ are endogenous parameters that will be determined in equilibrium. Note that $\tilde{x}_{i}+\phi_{y} \tilde{y}_{i}+\phi_{\omega} \tilde{\omega}>g$ is equivalent to $\frac{\tilde{\varepsilon}_{x, i}+\phi_{y} \tilde{\varepsilon}_{y, i}}{\sqrt{\tau_{x}^{-1}+\phi_{y}^{2} \tau_{y}^{-1}}}>\frac{g-\left(\tilde{a}+\phi_{y} \tilde{f}\right)-\phi_{\omega} \tilde{\omega}}{\sqrt{\tau_{x}^{-1}+\phi_{y}^{2} \tau_{y}^{-1}}}$, and hence speculators' aggregate purchase can be characterized by $1-\Phi\left(\frac{g-\left(\tilde{a}+\phi_{y} \tilde{f}\right)-\phi_{\omega} \tilde{\omega}}{\sqrt{\tau_{x}^{-1}+\phi_{y}^{2} \tau_{y}^{-1}}}\right)$. Similarly, their aggregate selling is $\Phi\left(\frac{g-\left(\tilde{a}+\phi_{y} \tilde{f}\right)-\phi_{\omega} \tilde{\omega}}{\sqrt{\tau_{x}^{-1}+\phi_{y}^{2} \tau_{y}^{-1}}}\right)$. Thus, the net holding from speculators is:

$$
D(\tilde{a}, \tilde{f}, \tilde{\omega})=1-2 \Phi\left(\frac{g-\left(\tilde{a}+\phi_{y} \tilde{f}\right)-\phi_{\omega} \tilde{\omega}}{\sqrt{\tau_{x}^{-1}+\phi_{y}^{2} \tau_{y}^{-1}}}\right) .
$$

The market clearing condition (5) together with equations (4) and (7) indicate that

$$
1-2 \Phi\left(\frac{g-\left(\tilde{a}+\phi_{y} \tilde{f}\right)-\phi_{\omega} \tilde{\omega}}{\sqrt{\tau_{x}^{-1}+\phi_{y}^{2} \tau_{y}^{-1}}}\right)=1-2 \Phi(\tilde{\xi}-\lambda \log \tilde{P}),
$$

which implies that the equilibrium price is given by:

$$
\tilde{P}=\exp \left(\frac{\tilde{a}}{\lambda \sqrt{\tau_{x}^{-1}+\phi_{y}^{2} \tau_{y}^{-1}}}+\frac{\phi_{y} \tilde{f}}{\lambda \sqrt{\tau_{x}^{-1}+\phi_{y}^{2} \tau_{y}^{-1}}}+\frac{\tilde{\xi}}{\lambda}+\frac{\phi_{\omega} \tilde{\omega}}{\lambda \sqrt{\tau_{x}^{-1}+\phi_{y}^{2} \tau_{y}^{-1}}}-\frac{g}{\lambda \sqrt{\tau_{x}^{-1}+\phi_{y}^{2} \tau_{y}^{-1}}}\right) .
$$

Recall that capital providers have the information set $\{\tilde{a}, \tilde{P}, \tilde{\omega}\}$, and so they know the realizations of $\tilde{a}$ and $\tilde{\omega}$. As a result, the price $\tilde{P}$ is equivalent to the following signal in predicting the productivity factor $\tilde{f}$ :

$$
\tilde{s}_{p} \equiv \frac{\lambda \sqrt{\tau_{x}^{-1}+\phi_{y}^{2} \tau_{y}^{-1}} \log \tilde{P}-\tilde{a}-\phi_{\omega} \tilde{\omega}+g}{\phi_{y}}=\tilde{f}+\tilde{\varepsilon}_{p},
$$

where

which has a precision of

$$
\tilde{\varepsilon}_{p} \equiv \frac{\sqrt{\tau_{x}^{-1}+\phi_{y}^{2} \tau_{y}^{-1}}}{\phi_{y}} \tilde{\xi}
$$

$$
\tau_{p} \equiv \frac{1}{\operatorname{Var}\left(\tilde{\varepsilon}_{p}\right)}=\frac{\phi_{y}^{2} \tau_{x} \tau_{y} \tau_{\xi}}{\tau_{y}+\phi_{y}^{2} \tau_{x}} .
$$

The endogenous precision $\tau_{p}$ captures how much information capital providers can learn from the price. It is crucially related to real efficiency through guiding capital providers' 
investment decisions. The public information precision $\tau_{\omega}$ affects $\tau_{p}$ only through its effect on $\phi_{y}$. Specifically, if speculators trade more aggressively on their information about $\tilde{f}$ (i.e., when $\phi_{y}$ becomes higher), the price is more informative about the factor $\tilde{f}$, all other things being equal. As a result, capital providers can glean more information from the price.

\subsubsection{The Role of the Feature of Two-Dimensional Uncertainty}

The mechanics of capital providers extracting information from the prices is also useful to illustrate the unique role of the feature of the two-factor uncertainty in delivering our results. Suppose we shut down the uncertainty related to factor $\tilde{a}$ by letting $\tau_{a}$ approach infinity, so that $\tilde{a}$ becomes common knowledge. Then, speculators will no longer rely on their signals $\tilde{x}_{i}$ in forming their trading strategies. We thus conjecture that speculators buy the asset whenever $\tilde{y}_{i}+\phi_{\omega}^{1 \operatorname{dim}} \tilde{\omega}>g^{1 \operatorname{dim}}$, where $\phi_{\omega}^{1 \operatorname{dim}}$ and $g^{1 \operatorname{dim}}$ are endogenous parameters. We can follow similar steps as above and show that speculators' aggregate demand for the risky asset is $D^{1 \operatorname{dim}}(\tilde{f}, \tilde{\omega})=1-2 \Phi\left(\frac{g^{1 \operatorname{dim}}-\tilde{f}-\phi_{\omega}^{1} \operatorname{dim} \tilde{\omega}}{\sqrt{\tau_{y}^{-1}}}\right)$. So, using market clearing condition (5), we can find that the equilibrium price would change to:

$$
\tilde{P}^{1 \operatorname{dim}}=\exp \left(\frac{\tilde{f}}{\lambda \sqrt{\tau_{y}^{-1}}}+\frac{\tilde{\xi}}{\lambda}-\frac{g^{1 \operatorname{dim}}}{\lambda \sqrt{\tau_{y}^{-1}}}+\frac{\phi_{\omega}^{1 \operatorname{dim}} \tilde{\omega}}{\lambda \sqrt{\tau_{y}^{-1}}}\right) .
$$

Given that capital providers know the public information $\tilde{\omega}$, the price $\tilde{P}^{1 \operatorname{dim}}$ is equivalent to the following signal in predicting $\tilde{f}$ :

$$
\tilde{s}_{p}^{1 \operatorname{dim}}=\tilde{f}+\sqrt{\tau_{y}^{-1}} \tilde{\xi},
$$

which has a precision of

$$
\tau_{p}^{1 \operatorname{dim}} \equiv \frac{1}{\operatorname{Var}\left(\sqrt{\tau_{y}^{-1} \tilde{\xi}}\right)}=\tau_{y} \tau_{\xi} .
$$

Clearly, the amount $\tau_{p}^{1 \mathrm{dim}}$ of information that capital providers learn from the price is not affected by the public information precision $\tau_{\omega}$, which shuts down the mechanism emphasized in our analysis. 


\subsection{The Optimal Investment Policy of Capital Providers}

Capital providers have information set $\mathcal{I}_{R}=\{\tilde{a}, \tilde{P}, \tilde{\omega}\}$. We have already characterized how they use the price $\tilde{P}$ to form a signal $\tilde{s}_{p}$ in predicting the factor $\tilde{f}$. Regarding the public signal $\tilde{\omega}$ in (2), they can use their knowledge of $\tilde{a}$ to transform $\tilde{\omega}$ into the following signal in predicting $\tilde{f}$ :

$$
\tilde{s}_{\omega} \equiv \frac{\tilde{\omega}-\mu_{a} \tilde{a}}{\mu_{f}}=\tilde{f}+\mu_{f}^{-1} \tilde{\varepsilon}_{\omega},
$$

which has a precision of $\mu_{f}^{2} \tau_{\omega}$. That is, capital providers' information set is equivalent to:

$$
\mathcal{I}_{R}=\left\{\tilde{a}, \tilde{s}_{p}, \tilde{s}_{\omega}\right\},
$$

where the two signals $\tilde{s}_{p}$ and $\tilde{s}_{\omega}$ are useful for predicting $\tilde{f}$.

By Bayes' rule and equation (1), we can compute that capital providers' optimal investment is:

$$
k_{j}^{*}=\exp \left[\left(\log \frac{\beta}{c}+\frac{1}{2} \frac{1}{\tau_{f}+\mu_{f}^{2} \tau_{\omega}+\tau_{p}}\right)+\tilde{a}+\frac{\mu_{f}^{2} \tau_{\omega}}{\tau_{f}+\mu_{f}^{2} \tau_{\omega}+\tau_{p}} \tilde{s}_{\omega}+\frac{\tau_{p}}{\tau_{f}+\mu_{f}^{2} \tau_{\omega}+\tau_{p}} \tilde{s}_{p}\right] .
$$

\subsection{The Optimal Trading Strategy of Speculators}

Using the expression of $\tilde{P}$ in (8), the cash flow expression $\tilde{V}=(1-\beta) \tilde{A} \tilde{F} K$, and the investment rule in (11), we can compute the expected price and cash flow conditional on speculator $i$ 's information set $\left\{\tilde{x}_{i}, \tilde{y}_{i}, \tilde{\omega}\right\}$ as follows:

$$
\begin{aligned}
& E\left(\tilde{P} \mid \tilde{x}_{i}, \tilde{y}_{i}, \tilde{\omega}\right)=\exp \left(b_{0}^{p}+b_{x}^{p} \tilde{x}_{i}+b_{y}^{p} \tilde{y}_{i}+b_{\omega}^{p} \tilde{\omega}\right), \\
& E\left(\tilde{V} \mid \tilde{x}_{i}, \tilde{y}_{i}, \tilde{\omega}\right)=\exp \left(b_{0}^{v}+b_{x}^{v} \tilde{x}_{i}+b_{y}^{v} \tilde{y}_{i}+b_{\omega}^{v} \tilde{\omega}\right),
\end{aligned}
$$

where the coefficients $b$ 's are given in the appendix.

Speculator $i$ will choose to buy the asset if and only if $E\left(\tilde{V} \mid \tilde{x}_{i}, \tilde{y}_{i}, \tilde{\omega}\right)>E\left(\tilde{P} \mid \tilde{x}_{i}, \tilde{y}_{i}, \tilde{\omega}\right)$. Thus, we have:

$$
\begin{aligned}
E\left(\tilde{V} \mid \tilde{x}_{i}, \tilde{y}_{i}, \tilde{\omega}\right) & >E\left(\tilde{P} \mid \tilde{x}_{i}, \tilde{y}_{i}, \tilde{\omega}\right) \Leftrightarrow \\
\left(b_{x}^{v}-b_{x}^{p}\right) \tilde{x}_{i}+\left(b_{y}^{v}-b_{y}^{p}\right) \tilde{y}_{i}+\left(b_{\omega}^{v}-b_{\omega}^{p}\right) \tilde{\omega} & >\left(b_{0}^{p}-b_{0}^{v}\right)-\log (1-\beta) .
\end{aligned}
$$

Recall that we conjecture speculators' trading strategy as buying the asset whenever $\tilde{x}_{i}+$ 
$\phi_{y} \tilde{y}_{i}+\phi_{\omega} \tilde{\omega}>g$. So, to be consistent with our initial conjecture, we require that in equilibrium,

$$
\begin{aligned}
\phi_{y} & =\frac{b_{y}^{v}-b_{y}^{p}}{b_{x}^{v}-b_{x}^{p}}, \\
\phi_{\omega} & =\frac{b_{\omega}^{v}-b_{\omega}^{p}}{b_{x}^{v}-b_{x}^{p}} .
\end{aligned}
$$

The right-hand-side $\frac{b_{y}^{v}-b_{y}^{p}}{b_{x}^{v}-b_{x}^{p}}$ of (14) depends only on $\phi_{y}$ (through the term of $\phi_{y}$ in $b_{y}^{p}$ and $b_{x}^{p}$ and the term of $\tau_{p}$ in $b_{v}^{p}$ and $b_{v}^{p}$ ). Therefore, we can use (14) to compute $\phi_{y}$, and then plug this solved $\phi_{y}$ into equation (15) to compute $\phi_{\omega}$.

We have the following characterization proposition.

Proposition 1 The equilibrium is characterized by the weight $\phi_{y}>0$ that speculators put on the private signal $\tilde{y}_{i}$ about factor $\tilde{f}$, and $\phi_{y}$ is determined by condition (14).

\section{Good Disclosure, Bad Disclosure}

\subsection{Real Efficiency and Public Information}

In this section we study the implications of disclosure for real efficiency. We follow Goldstein, Ozdenoren, and Yuan (2013) and measure real efficiency by the ex-ante expected net benefit of investment evaluated at equilibrium. We can compute

$$
\begin{aligned}
R E & \equiv E\left[\tilde{A} \tilde{F} K-\int c\left(k_{j}\right) d j\right] \\
& =\frac{\beta}{c}\left(1-\frac{\beta}{2}\right) \exp \left[\frac{2}{\tau_{a}}+\frac{2}{\tau_{f}}-\operatorname{Var}\left(\tilde{f} \mid \tilde{s}_{\omega}, \tilde{s}_{p}\right)\right],
\end{aligned}
$$

where

$$
\operatorname{Var}\left(\tilde{f} \mid \tilde{s}_{\omega}, \tilde{s}_{p}\right)=\frac{1}{\tau_{f}+\mu_{f}^{2} \tau_{\omega}+\tau_{p}} .
$$

In our model, disclosure affects real efficiency through changing capital providers' information set. The more information that capital providers have, the better are their investment decisions, and so is the real efficiency in aggregate. This fact is captured by the term $\operatorname{Var}\left(\tilde{f} \mid \tilde{s}_{\omega}, \tilde{s}_{p}\right)$ in (16).

Equation (17) demonstrates that public information has two effects on capital providers' information set (and hence real efficiency). The first is a direct effect of providing new information, i.e., the term $\mu_{f}^{2} \tau_{\omega}$ in (17). The second effect is an endogenous indirect effect: 
Public information affects the trading of speculators (more specifically, the loading $\phi_{y}$ on the private information about $\tilde{f}$ ), and hence the price informativeness about the factor $\tilde{f}$, which in turn affects the amount of information that capital providers can learn from the price (i.e., the term $\tau_{p}$ in (17)). Formally, by (16) and (17), we have:

$$
\frac{\partial R E}{\partial \tau_{\omega}} \propto \frac{\partial\left(\tau_{f}+\mu_{f}^{2} \tau_{\omega}+\tau_{p}\right)}{\partial \tau_{\omega}}=\mu_{f}^{2}+\frac{\partial \tau_{p}}{\partial \tau_{\omega}},
$$

where the term $\mu_{f}^{2}$ captures disclosure's direct effect, and where by (10),

$$
\frac{\partial \tau_{p}}{\partial \tau_{\omega}}=\frac{2 \tau_{p} \tau_{y}}{\phi_{y}\left(\tau_{y}+\phi_{y}^{2} \tau_{x}\right)} \frac{\partial \phi_{y}}{\partial \tau_{\omega}},
$$

which captures disclosure's indirect effect through affecting the trading strategy $\phi_{y}$.

In the following two subsections, we consider two types of public information - one about factor $\tilde{a}$ and the other about factor $\tilde{f}$. We show that releasing public information about $\tilde{a}$ is always beneficial to real efficiency, while the effect of releasing public information about $\tilde{f}$ is ambiguous. More specifically, disclosing information about $\tilde{f}$ harms (improves) real efficiency if the market aggregates speculators' private information effectively (ineffectively).

\subsection{The Effect of Disclosure about Factor $\tilde{a}$}

As we discussed before, when $\frac{\operatorname{Var}\left(\mu_{a} \tilde{a}\right)}{\operatorname{Var}\left(\mu_{f} \tilde{f}\right)}$ is very large, the public signal $\tilde{\omega}$ in $(2)$ is primarily a signal about $\tilde{a}$. For simplicity, we assume $\mu_{f}=0$ and normalize $\mu_{a}$ as 1 , so that $\tilde{\omega}$ degenerates to

$$
\tilde{\omega}=\tilde{a}+\tilde{\varepsilon}_{\omega}
$$

In this case, since capital providers know $\tilde{a}$ perfectly, the direct effect of public disclosure vanishes (i.e., $\frac{\partial \mu_{f}^{2} \tau_{\omega}}{\partial \tau_{\omega}}=\mu_{f}^{2}=0$ in (18)). Therefore, the only effect for public disclosure to affect real efficiency is through its indirect effect on the endogenous precision of the information that capital providers can learn from the asset price (i.e., $\frac{\partial R E}{\partial \tau_{\omega}} \propto \frac{\partial \tau_{p}}{\partial \tau_{\omega}}$ in (18)).

We can compute that the terms $b$ 's in (14) are as follows:

$$
\begin{aligned}
b_{x}^{p} & =\frac{\frac{\tau_{x}}{\tau_{a}+\tau_{x}+\tau_{\omega}}}{\lambda \sqrt{\tau_{x}^{-1}+\phi_{y}^{2} \tau_{y}^{-1}}}, b_{y}^{p}=\frac{\frac{\phi_{y} \tau_{y}}{\tau_{f}+\tau_{y}}}{\lambda \sqrt{\tau_{x}^{-1}+\phi_{y}^{2} \tau_{y}^{-1}}}, \\
b_{x}^{v} & =\frac{2 \tau_{x}}{\tau_{a}+\tau_{x}+\tau_{\omega}}, b_{y}^{v}=\left(1+\frac{\tau_{p}}{\tau_{f}+\tau_{p}}\right) \frac{\tau_{y}}{\tau_{f}+\tau_{y}} .
\end{aligned}
$$


Thus, as the supply elasticity $\lambda$ becomes large, $b_{x}^{p}$ and $b_{y}^{p}$ approach to 0 , and thus the condition of (14) determining $\phi_{y}$ degenerates to:

$$
\phi_{y} \approx \frac{b_{y}^{v}}{b_{x}^{v}}=\frac{\left(1+\frac{\tau_{p}}{\tau_{f}+\tau_{p}}\right) \frac{\tau_{y}}{\tau_{f}+\tau_{y}}}{\frac{2 \tau_{x}}{\tau_{a}+\tau_{x}+\tau_{\omega}}} .
$$

The above condition makes sense. Recall that speculators buy the asset whenever $\tilde{x}_{i}+$ $\phi_{y} \tilde{y}_{i}+\phi_{\omega} \tilde{\omega}>g$, and so the sensitivity $\phi_{y}$ captures how aggressively they trade on their private information $\tilde{y}_{i}$ relative to their private information $\tilde{x}_{i}$. When $\lambda$ is large, the price is highly elastic, so that the price is not much affected by speculators' trade (See equation (8)). As a consequence, speculators will trade mainly based on their expectations $E\left(\tilde{V} \mid \tilde{x}_{i}, \tilde{y}_{i}, \tilde{\omega}\right)$ in (13) about the asset's future cash flow. It is therefore reasonable that $\phi_{y}$ is mainly determined by the ratio $\frac{b_{y}^{v}}{b_{x}^{v}}$ of the two coefficients that speculators put on signals $\tilde{y}_{i}$ and $\tilde{x}_{i}$, respectively, in forecasting the future value of the asset.

Using the expression of $\tau_{p}$ in (10) and applying the implicit function theorem to (20), we can show:

$$
\frac{\partial \phi_{y}}{\partial \tau_{\omega}}=\frac{\frac{\phi_{y}}{\tau_{a}+\tau_{x}+\tau_{\omega}}}{1-\frac{2 \tau_{p} \tau_{y} \tau_{f}}{\left(\tau_{f}+\tau_{p}\right)\left(\tau_{f}+2 \tau_{p}\right)\left(\tau_{y}+\phi_{y}^{2} \tau_{x}\right)}}>0 ;
$$

that is, public disclosure about factor $\tilde{a}$ causes speculators to trade more aggressively on their private information about the other factor $\tilde{f}$.

The intuition for this result lies in condition (20). Note that in the expression of $E\left(\tilde{V} \mid \tilde{x}_{i}, \tilde{y}_{i}, \tilde{\omega}\right)$ in $(13)$, the private signal $\tilde{x}_{i}$ and the public signal $\tilde{\omega}$ are useful in predicting $\tilde{a}$, and the private signal $\tilde{y}_{i}$ is useful in predicting $\tilde{f}$. When $\tau_{\omega}$ increases, so that the public signal becomes a more informative signal about $\tilde{a}$, speculators will put a higher weight $b_{\omega}^{v}$ on the signal $\tilde{\omega}$ and a lower weight $b_{x}^{v}$ on the signal $\tilde{x}_{i}$ in predicting $\tilde{a}$. Other things being equal, this will increase $\phi_{y}$ since $\phi_{y}=\frac{b_{y}^{v}}{b_{x}^{v}}$ in (20). However, this is not the end of the story, because there is a further "multiplier effect" (as captured by the denominator in (21)): The increased $\phi_{y}$ will improve $\tau_{p}$ in (10), and so capital providers will collect more information on $\tilde{f}$ from the price, making the asset cash flow $\tilde{V}$ more responsive to $\tilde{f}$ through capital providers' investments; this will in turn cause speculators to rely more on their private private $\tilde{y}_{i}$ - which is a signal about $\tilde{f}$ - in making their forecasts, which increases $b_{y}^{v}$ in (13). So, $\phi_{y}$ will continue to increase through (20). This amplification chain continues on 
and on till it converges to a much higher level of $\phi_{y}$.

Since $\frac{\partial \phi_{y}}{\partial \tau_{\omega}}>0$ by $(21)$, we have $\frac{\partial \tau_{p}}{\partial \tau_{\omega}}>0$ as well in (19). That is, capital providers learn more information from the price. Thus, real efficiency will improve with disclosure, since when the public signal communicates information only about $\tilde{a}$, it affects real efficiency only through its effect on the price informativeness about $\tilde{f}$.

By continuity, all the above results for the case of $\mu_{a}=1$ and $\mu_{f}=0$ will go through for cases with $\frac{\operatorname{Var}\left(\mu_{a} \tilde{a}\right)}{\operatorname{Var}\left(\mu_{f} \tilde{f}\right)}$ close to $\infty$. To summarize, we have the following proposition.

Proposition 2 Suppose that the supply elasticity $\lambda$ is high and that the public information is mainly a signal about factor $\tilde{a}$ (i.e., $\frac{\operatorname{Var}\left(\mu_{a} \tilde{a}\right)}{\operatorname{Var}\left(\mu_{f} \tilde{f}\right)}$ is large). Then,

(a) there exists an equilibrium, where the relative weight $\phi_{y}>0$ on private signals $\tilde{y}_{i}$ about the other factor $\tilde{f}$ in speculators' trading strategy is determined by (20);

(b) increasing the precision $\tau_{\omega}$ of public disclosure

(i) increases the relative weight $\phi_{y}>0$ on private signals $\tilde{y}_{i}$ (i.e., $\frac{\partial \phi_{y}}{\partial \tau_{\omega}}>0$ );

(ii) increases the precision $\tau_{p}$ that capital providers learn from the price regarding the factor $\tilde{f}\left(i . e ., \frac{\partial \tau_{p}}{\partial \tau_{\omega}}>0\right)$; and

(iii) increases the real investment efficiency $R E$ (i.e., $\frac{\partial R E}{\partial \tau_{\omega}}>0$ ).

Figure 1 graphically illustrates Proposition 2. Here, we simply set the precision of all random variables) to be 1 ; that is, $\tau_{a}=\tau_{f}=\tau_{x}=\tau_{y}=\tau_{\xi}=1$. The patterns are quite robust with respect to changes in these precision parameter values. We also choose $\mu_{a}=0.8$ and $\mu_{f}=0.2$, so that the public signal $\tilde{\omega}$ is mainly a signal about factor $\tilde{a}$. Note that under this parameter configuration, $\tilde{\omega}$ also provides information about factor $\tilde{f}$. In Panel (a), we plot the weight $\phi_{y}$ that speculators put on the private signal $\tilde{y}_{i}$ against the precision $\tau_{\omega}$ of the public signal. In Panel (b), we plot three variables against $\tau_{\omega}$ : (1) $\mu_{f}^{2} \tau_{\omega}$, the direct effect of public disclosure on capital providers' forecast precision by providing new information about $\tilde{f} ;(2) \tau_{p}$, the indirect effect of public disclosure on capital providers' forecast precision by affecting the informational content in the price; and (3) $\mu_{f}^{2} \tau_{\omega}+\tau_{p}$, which is a proxy for real efficiency, since by (16) and (17), real efficiency $R E$ is a monotonic transformation of $\mu_{f}^{2} \tau_{\omega}+\tau_{p}$

[INSERT FIGURE 1 HERE.] 
We see that in Panel (a), as Proposition 2 predicts, increasing the precision $\tau_{\omega}$ of the public signal increases the weight $\phi_{y}$ that speculators put on the private signal $\tilde{y}_{i}$ about factor $\tilde{f}$. This in turn means that in Panel (b), the precision $\tau_{p}$ of information that capital providers learn from the price increases, because $\tau_{p}$ increases with $\phi_{y}$ by (10). Clearly, the direct effect $\mu_{f}^{2} \tau_{\omega}$ of disclosure increases with $\tau_{\omega}$ as well in Panel (b). As a result, the overall effect of disclosure is to increase real efficiency $\mu_{f}^{2} \tau_{\omega}+\tau_{p}$.

\subsection{The Effect of Disclosure about Factor $\tilde{f}$}

When $\frac{\operatorname{Var}\left(\mu_{a} \tilde{a}\right)}{\operatorname{Var}\left(\mu_{f} \tilde{f}\right)}$ is very small, the public signal $\tilde{\omega}$ in $(2)$ is primarily a signal about $\tilde{f}$. For simplicity, we assume $\mu_{a}=0$ and normalize $\mu_{f}$ as 1 , so that $\tilde{\omega}$ degenerates to

$$
\tilde{\omega}=\tilde{f}+\tilde{\varepsilon}_{\omega} .
$$

In this case, both effects of public disclosure are active in equation (18). First, the public signal $\tilde{\omega}$ directly benefits capital providers by providing information that they wish to learn. Second, it affects the trading behavior of speculators and hence the informational content in the price, thereby indirectly affecting capital providers' forecast.

We can compute that the terms $b$ 's in (14) are as follows:

$$
\begin{aligned}
b_{x}^{p} & =\frac{\frac{\tau_{x}}{\tau_{a}+\tau_{x}}}{\lambda \sqrt{\tau_{x}^{-1}+\phi_{y}^{2} \tau_{y}^{-1}}}, b_{y}^{p}=\frac{\frac{\phi_{y} \tau_{y}}{\tau_{f}+\tau_{y}+\tau_{\omega}}}{\lambda \sqrt{\tau_{x}^{-1}+\phi_{y}^{2} \tau_{y}^{-1}}}, \\
b_{x}^{v} & =\frac{2 \tau_{x}}{\tau_{a}+\tau_{x}}, b_{y}^{v}=\left(1+\frac{\tau_{p}}{\tau_{f}+\tau_{\omega}+\tau_{p}}\right) \frac{\tau_{y}}{\tau_{f}+\tau_{y}+\tau_{\omega}} .
\end{aligned}
$$

Thus, as the supply elasticity $\lambda$ becomes large, $b_{x}^{p}$ and $b_{y}^{p}$ approach to 0 , and thus the condition of (14) determining $\phi_{y}$ degenerates to:

$$
\phi_{y} \approx \frac{b_{y}^{v}}{b_{x}^{v}}=\frac{\frac{\tau_{y}\left(\tau_{f}+2 \tau_{p}+\tau_{\omega}\right)}{\left(\tau_{f}+\tau_{p}+\tau_{\omega}\right)\left(\tau_{f}+\tau_{y}+\tau_{\omega}\right)}}{\frac{2 \tau_{x}}{\tau_{a}+\tau_{x}}} .
$$

Applying the implicit function theorem to the above equation, we can show:

$$
\frac{\partial \phi_{y}}{\partial \tau_{\omega}}=-\frac{\phi_{y}\left(\frac{\tau_{p}}{\left(\tau_{f}+2 \tau_{p}+\tau_{\omega}\right)\left(\tau_{f}+\tau_{p}+\tau_{\omega}\right)}+\frac{1}{\tau_{f}+\tau_{y}+\tau_{\omega}}\right)}{1-\frac{2 \tau_{p} \tau_{y}\left(\tau_{f}+\tau_{\omega}\right)}{\left(\tau_{f}+\tau_{p}+\tau_{\omega}\right)\left(\tau_{f}+2 \tau_{p}+\tau_{\omega}\right)\left(\tau_{y}+\phi_{y}^{2} \tau_{x}\right)}}<0 ;
$$

that is, public disclosure about factor $\tilde{f}$ causes speculators to trade less aggressively on their own private information about $\tilde{f}$. 
Similar to the result for disclosure about $\tilde{a}$, the intuition here also lies in equation (22) that determines the equilibrium value of $\phi_{y}$. By $(22), \phi_{y}$ is determined by the ratio $\frac{b_{y}^{v}}{b_{x}^{v}}$. When the public information $\tilde{\omega}$ is mainly a signal about $\tilde{f}$, increasing its precision $\tau_{\omega}$ will decrease the weight $b_{y}^{v}$ on speculators' own private signal $\tilde{y}_{i}$ in predicting $\tilde{f}$. This tends to decrease $\phi_{y}$ through $\phi_{y}=\frac{b_{y}^{v}}{b_{x}^{v}}$ in (22). In addition, there is also a multiplier effect, as captured by the denominator in (23): The decreased $\phi_{y}$ reduces $\tau_{p}$, which will cause capital providers to collect less information about $\tilde{f}$, making the asset value $\tilde{V}$ less sensitive to $\tilde{f}$; so, speculators will trade more aggressively on their private information $\tilde{x}_{i}$ about the other factor $\tilde{a}$ and less aggressively on information $\tilde{y}_{i}$ about $\tilde{f}$ (that is, $b_{x}^{v}$ becomes higher and $b_{y}^{v}$ becomes lower); and this will further reduce $\phi_{y}$ through (22), until the equilibrium value $\phi_{y}$ reaches a much lower level.

By (10), the effect on $\tau_{p}$ of disclosure is still given by equation (19). Since $\frac{\partial \phi_{y}}{\partial \tau_{\omega}}<0$ by (23), we have $\frac{\partial \tau_{p}}{\partial \tau_{\omega}}<0$ as well. That is, as the precision of public information (about factor $\tilde{f}$ ) increases, capital providers learn less information from the price.

The effect of disclosure on real efficiency is ambiguous. On the one hand, public disclosure improves real efficiency by directly providing new information to capital providers; that is, $\frac{\partial \mu_{f}^{2} \tau_{\omega}}{\partial \tau_{\omega}}=\mu_{f}^{2}>0$ in (18). On the other hand, disclosure reduces the price informativeness $\tau_{p}$ about the factor $\tilde{f}$ that capital providers care to learn; that is, $\frac{\partial \tau_{p}}{\partial \tau_{\omega}}<0$ in (18). So, the overall real-efficiency effect depends on the relative strength of these two effects. We can show that when the precision $\tau_{\xi}$ is large (small), the negative indirect effect (positive direct effect) dominates. This makes sense: When $\tau_{\xi}$ is large, the market aggregates speculators' private information effectively, and so the indirect effect related to price informativeness is strong; by contrast, when $\tau_{\xi}$ is small, the market has a lot of noise trading, and its informationaggregation role is limited, weakening disclosure's indirect effect via price informativeness.

By continuity, all the above results for the case of $\mu_{a}=0$ and $\mu_{f}=1$ will go through for cases with $\frac{\operatorname{Var}\left(\mu_{a} \tilde{a}\right)}{\operatorname{Var}\left(\mu_{f} \tilde{f}\right)}$ close to 0 . To summarize, we have the following proposition (the formal proof for Part (b.iii) is provided in appendix).

Proposition 3 Suppose that the supply elasticity $\lambda$ is high and that the public information is mainly a signal about factor $\tilde{f}$ (i.e., $\frac{\operatorname{Var}\left(\mu_{a} \tilde{a}\right)}{\operatorname{Var}\left(\mu_{f} \tilde{f}\right)}$ is small). Then,

(a) there exists an equilibrium, where the relative weight $\phi_{y}>0$ on private signals $\tilde{y}_{i}$ about 
factor $\tilde{f}$ in speculators' trading strategy is determined by equation (22);

(b) increasing the precision $\tau_{\omega}$ of public disclosure

(i) decreases the relative weight $\phi_{y}$ on private signals $\tilde{y}_{i}$ (i.e., $\frac{\partial \phi_{y}}{\partial \tau_{\omega}}<0$ );

(ii) decreases the precision $\tau_{p}$ that capital providers learn from the price regarding the factor $\tilde{f}$ (i.e., $\left.\frac{\partial \tau_{p}}{\partial \tau_{\omega}}<0\right)$; and

(iii) decreases (increases) the real investment efficiency $R E$ if the precision $\tau_{\xi}$ of noise trading is large (small) (i.e., $\frac{\partial R E}{\partial \tau_{\omega}}<0$ if $\tau_{\xi}$ is large, and $\frac{\partial R E}{\partial \tau_{\omega}}>0$ if $\tau_{\xi}$ is small).

Figures 2 and 3 graphically illustrates Proposition 3. As in Figure 1, we set $\tau_{a}=\tau_{f}=$ $\tau_{x}=\tau_{y}=1$ and $\lambda=2$. In both figures, we set $\mu_{a}=0.2$ and $\mu_{f}=0.8$, so that the public information is primarily a signal about factor $\tilde{f}$. In Figure 2 , we choose $\tau_{\xi}=5$, so that the market aggregates private information effectively. In Figure 3, we choose $\tau_{\xi}=0.2$, and thus, the level $\frac{1}{\tau_{\xi}}$ of noise trading is relatively high and the market does not aggregate private information that much.

\section{[INSERT FIGURES 2 AND 3 HERE.]}

In Panel (a) of both figures, we see that, consistent with Proposition 3, the relative weight $\phi_{y}$ that speculators put on the private information $\tilde{y}_{i}$ decreases with the precision $\tau_{\omega}$ of public information. This translates to a decreasing $\tau_{p}$ as a function of $\tau_{\omega}$ in Panel (b) of both figures. Also, increasing $\tau_{\omega}$ directly improves capital providers' learning, as manifested by the increasing $\mu_{f}^{2} \tau_{\omega}$ in Panel (b) of both figures. However, in Figure 2 where $\tau_{\xi}$ is relatively large, the negative effect on $\tau_{p}$ dominates, so that overall the real efficiency $\left(\mu_{f}^{2} \tau_{\omega}+\tau_{p}\right)$ decreases with $\tau_{\omega}$. By contrast, in Figure 3 where $\tau_{\xi}$ is relatively small, the positive effect on $\mu_{f}^{2} \tau_{\omega}$ dominates, and the real efficiency $\left(\mu_{f}^{2} \tau_{\omega}+\tau_{p}\right)$ increases with $\tau_{\omega}$.

\section{An Extension: Capital Providers Receive Noisy Sig- nals about Factors}

In the model analyzed in previous sections, we have assumed that capital providers know factor $\tilde{a}$ perfectly and know nothing about factor $\tilde{f}$, so that they care only about the price's informational content about $\tilde{f}$. In this section, we extend our model by assuming that capital 
providers receive noisy signals about both factors, and show that all our results go through as long as capital providers wish to learn one productivity factor more than the other.

Specifically, we now endow each capital provider $j$ with two private signals

$$
\tilde{z}_{j}=\tilde{a}+\tilde{\varepsilon}_{z, j} \text { and } \tilde{s}_{j}=\tilde{f}+\tilde{\varepsilon}_{s, j}
$$

where $\tilde{\varepsilon}_{z, j} \sim N\left(0, \frac{1}{\tau_{z}}\right)$ (with $\tau_{z}>0$ ) and $\tilde{\varepsilon}_{s, j} \sim N\left(0, \frac{1}{\tau_{s}}\right)$ (with $\tau_{s}>0$ ) and they are independent of all other random variables. We keep intact all the other features of the model. Our baseline model corresponds to the case of $\tau_{z}=\infty$ and $\tau_{s}=0$. If $\frac{\tau_{z}}{\tau_{a}}$ and $\frac{\tau_{s}}{\tau_{f}}$ are sufficiently different, then capital providers are more keen to learn one factor more than the other.

We still consider trading strategies that speculators buy the asset if and only $\tilde{x}_{i}+\phi_{y} \tilde{y}_{i}+$ $\phi_{\omega} \tilde{\omega}>g$, where $\phi_{y}, \phi_{\omega}$, and $g$ are endogenous parameters determined in equilibrium. So, their aggregate demand $D(\tilde{a}, \tilde{f}, \tilde{\omega})$ is still given by equation $(7)$, and the market clearing condition still implies a price function $P(\tilde{a}, \tilde{f}, \tilde{\omega}, \tilde{\xi})$ in equation (8). However, because now capital providers do not observe $\tilde{a}$ perfectly, the price is no longer a signal about $\tilde{f}$ given by (9); but instead, it is a signal about both $\tilde{a}$ and $\tilde{f}$ as follows:

$$
\tilde{s}_{p}^{e x t}=\frac{\tilde{a}}{\phi_{y}}+\tilde{f}+\tilde{\varepsilon}_{p}
$$

where $\tilde{\varepsilon}_{p}$ is still defined by $\tilde{\varepsilon}_{p} \equiv \frac{\sqrt{\tau_{x}^{-1}+\phi_{y}^{2} \tau_{y}^{-1}}}{\phi_{y}} \tilde{\xi}$.

Each capital provider $j$ 's optimal investment decision is:

$$
k_{j}^{*}=\arg \max _{k_{j}} E\left(\beta \tilde{A} \tilde{F} k_{j}-\frac{c}{2} k_{j}^{2} \mid \tilde{z}_{j}, \tilde{s}_{j}, \tilde{\omega}, \tilde{s}_{p}^{e x t}\right)=\frac{\beta E\left(e^{\tilde{a}+\tilde{f}} \mid \tilde{z}_{j}, \tilde{s}_{j}, \tilde{\omega}, \tilde{s}_{p}^{e x t}\right)}{c} .
$$

We can still show that capital providers follow a loglinear investment rule:

$$
k_{j}^{*}=\exp \left(h_{0}+h_{z} \tilde{z}_{j}+h_{s} \tilde{s}_{j}+h_{\omega} \tilde{\omega}+h_{p} \tilde{s}_{p}^{e x t}\right),
$$

where $h$ 's are endogenous constants that depend on $\left(\tau_{a}, \tau_{f}, \tau_{z}, \tau_{s}, \mu_{a}, \mu_{f}, \phi_{y}, \tau_{p}\right)$. We then follow steps similar to the baseline model and show that the characterization of the equilibrium boils down to one equation in terms of the loading $\phi_{y}$ that speculators put on their private signals $\tilde{y}_{i}$. The complexity of the inference problem induced by the price signal $\tilde{s}_{p}^{e x t}$ in (24) precludes a full analytical characterization of the equilibrium, and therefore we rely 
on numerical analysis.

\section{[INSERT FIGURE 4 HERE.]}

In the right panels of Figure 4, we plot real efficiency, $R E=E\left[\tilde{A} \tilde{F} K-\int c\left(k_{j}\right) d j\right]$, against the precision $\tau_{\omega}$ of public information. In the left panels, we also plot the direct and indirect effects of public information on the inference problem of capital providers. Specifically, in the first-order-condition (25) of capital providers' decision problem, they wish to forecast the total productivity $\tilde{a}+\tilde{f}$ using information $\left\{\tilde{z}_{j}, \tilde{s}_{j}, \tilde{\omega}, \tilde{s}_{p}^{e x t}\right\}$. Note that capital providers have private information $\left\{\tilde{z}_{j}, \tilde{s}_{j}\right\}$, and the forecast precision given their own information is $\frac{1}{\operatorname{Var}\left(\tilde{a}+\tilde{f} \mid \tilde{z}_{j}, \tilde{s}_{j}\right)}$. After adding the public information, their forecast precision increases to $\frac{1}{\operatorname{Var}\left(\tilde{a}+\tilde{f} \mid \tilde{z}_{j}, \tilde{s}_{j}, \tilde{\omega}\right)}$, and so we can use the difference of $\frac{1}{\operatorname{Var}\left(\tilde{a}+\tilde{f} \mid \tilde{z}_{j}, \tilde{s}_{j}, \tilde{\omega}\right)}-\frac{1}{\operatorname{Var}\left(\tilde{a}+\tilde{f} \mid \tilde{z}_{j}, \tilde{s}_{j}\right)}$ to measure the direct effect of disclosure. This is consistent with using $\mu_{f}^{2} \tau_{\omega}\left(=\frac{1}{\operatorname{Var}(\tilde{a}+\tilde{f} \mid \tilde{a} \tilde{\omega})}-\right.$ $\left.-\frac{1}{\operatorname{Var}(\tilde{a}+\tilde{f} \mid \tilde{a})}\right)$ to measure the direct effect in our baseline model. Similarly, we will use the difference $\frac{1}{\operatorname{Var}\left(\tilde{a}+\tilde{f} \mid \tilde{z}_{j}, \tilde{s}_{j}, \tilde{\omega}, \tilde{s}_{p}^{e x t}\right)}-\frac{1}{\operatorname{Var}\left(\tilde{a}+\tilde{f} \mid \tilde{z}_{j}, \tilde{s}_{j}, \tilde{\omega}\right)}$ to capture how disclosure indirectly affects capital providers' forecast through affecting the informational content of the asset price.

In all panels of Figure 4, we set $\tau_{a}=\tau_{f}=\tau_{x}=\tau_{y}=1, \beta=\frac{1}{2}, c=1$, and $\gamma=2$. We also choose $\tau_{z}=5$ and $\tau_{s}=1$; that is, capital providers know more about factor $\tilde{a}$ than factor $\tilde{f}$. In Panels (a1)-(a2), we set $\mu_{a}=0.8$ and $\mu_{f}=0.2$, so that the public information $\tilde{\omega}$ is primarily a signal about factor $\tilde{a}$. We also arbitrarily choose $\tau_{\xi}=1$ in these two panels. In contrast, in the remaining four panels (Panels (b1)-(c2)), we set $\mu_{a}=0.2$ and $\mu_{f}=0.8$, so that the public information $\tilde{\omega}$ is primarily a signal about factor $\tilde{f}$. Also, in Panels (b1)-(b2), we choose $\tau_{\xi}=5$ to make the market aggregate private information effectively, and in Panels (c1)-(c2), we choose $\tau_{\xi}=0.2$, so that the market doe not aggregate private information that much.

We see that Panels (a1)-(a2) of Figure 4 deliver the same message as Figure 1: When the pubic information is mainly a signal about $\tilde{a}$, increasing the precision of public disclosure both directly and indirectly benefit capital providers' learning, which in turn improves real efficiency. Panels (b1)-(b2) are also consistent with Figure 2: When the public information is mainly a signal about $\tilde{f}$, public disclosure directly improves but indirectly harms capital providers' learning, and when the market aggregates speculators' private information effec- 
tively, the indirect effect dominates, so that the overall effect is that real efficiency decreases with disclosure precision. Finally, Panels (c1)-(c2) are in line with Figure 3: When the public information is mainly a signal about $\tilde{f}$ and when the market does not aggregate speculators' private information effectively, the positive direct effect of public disclosure dominates and the overall effect of disclosure is to improve real efficiency.

\section{Conclusion}

Public disclosure has been an important component of financial regulation. Various agencies, corporate companies and governments, for example, frequently make public announcements to the financial market. The financial market is not a just sideshow, because the market price conveys valuable information relevant for real decision makers, such as managers and capital providers, to make decisions. Moreover, the fluctuations of asset price are driven by multiple factors, and real decision makers care to learn some factors more than others. In this paper, we have proposed a framework to study what kind of public information is "good" in the sense that it improves the forecast quality of real decision makers and thus real efficiency.

We find that public information release generally has two effects on real efficiency. First, it benefits real decision makers' forecast problem by directly providing new information. Second, it can benefit or harm real decision makers' learning indirectly by affecting the informational content of asset price. When the public disclosure is mainly a signal about something of which real decision makers are already well informed, both the direct and indirect effects are positive, and thus public information improves real efficiency. In contrast, when the public disclosure mainly conveys information that real decision makers care to learn much, then the direct effect is generally negative, and when the financial market is a very effective device of aggregating traders' private information, this negative indirect effect dominates, so that public disclosure actually harms real efficiency. 


\section{Appendix}

\section{The Expressions of the Coefficients b's in Equations (12) and (13)}

Define $\Delta^{p} \equiv \operatorname{Var}\left(\tilde{a}+\phi_{y} \tilde{f} \mid \tilde{x}_{i}, \tilde{y}_{i}, \tilde{\omega}\right)$, and let $\delta_{x}^{p}, \delta_{y}^{p}$, and $\delta_{\omega}^{p}$ be the loadings of $\tilde{x}_{i}, \tilde{y}_{i}$ and $\tilde{\omega}$ in the expression of $E\left(\tilde{a}+\phi_{y} \tilde{f} \mid \tilde{x}_{i}, \tilde{y}_{i}, \tilde{\omega}\right)$, respectively. Then, we have:

$$
\begin{aligned}
b_{0}^{p} & =\frac{-g}{\lambda \sqrt{\tau_{x}^{-1}+\phi_{y}^{2} \tau_{y}^{-1}}}+\frac{1}{2 \lambda^{2} \tau_{\xi}}+\frac{\Delta^{p}}{2 \lambda^{2}\left(\tau_{x}^{-1}+\phi_{y}^{2} \tau_{y}^{-1}\right)} \\
b_{x}^{p} & =\frac{\delta_{x}^{p}}{\lambda \sqrt{\tau_{x}^{-1}+\phi_{y}^{2} \tau_{y}^{-1}}}, b_{y}^{p}=\frac{\delta_{y}^{p}}{\lambda \sqrt{\tau_{x}^{-1}+\phi_{y}^{2} \tau_{y}^{-1}}}, b_{\omega}^{p}=\frac{\delta_{\omega}^{p}+\phi_{\omega}}{\lambda \sqrt{\tau_{x}^{-1}+\phi_{y}^{2} \tau_{y}^{-1}}} .
\end{aligned}
$$

Similarly, define $\Delta^{v} \equiv \operatorname{Var}\left(\tilde{a}+\frac{1+\frac{\tau_{p}}{\tau_{f}+\mu_{f}^{2} \tau_{\omega}+\tau_{p}}}{2-\frac{\mu_{f}^{2} \tau_{\omega}}{\tau_{f}+\mu_{f}^{2} \tau_{\omega}+\tau_{p}} \frac{\mu_{a}}{\mu_{f}}} \tilde{f} \mid \tilde{x}_{i}, \tilde{y}_{i}, \tilde{\omega}\right)$, and let $\delta_{x}^{v}, \delta_{y}^{v}$, and $\delta_{\omega}^{v}$ be the loadings of $\tilde{x}_{i}, \tilde{y}_{i}$ and $\tilde{\omega}$ in the expression of $E\left(\tilde{a}+\frac{1+\frac{\tau_{p}}{\tau_{f}+\mu_{f}^{2} \tau_{\omega}+\tau_{p}}}{2-\frac{\mu_{f}^{2} \tau_{\omega}}{\tau_{f}+\mu_{f}^{2} \tau_{\omega}+\tau_{p}} \frac{\mu_{a}}{\mu_{f}}} \tilde{f} \mid \tilde{x}_{i}, \tilde{y}_{i}, \tilde{\omega}\right)$, respectively. Then, we have:

$$
\begin{aligned}
b_{0}^{v} & =\log \frac{\beta(1-\beta)}{c}+\frac{\tau_{p}\left(\tau_{f}+\mu_{f}^{2} \tau_{\omega}+\tau_{p}\right)+1}{2 \tau_{p}\left(\tau_{f}+\mu_{f}^{2} \tau_{\omega}+\tau_{p}\right)^{2}}+\frac{\Delta^{v}}{2}\left(2-\frac{\mu_{f}^{2} \tau_{\omega}}{\tau_{f}+\mu_{f}^{2} \tau_{\omega}+\tau_{p}} \frac{\mu_{a}}{\mu_{f}}\right)^{2}, \\
b_{x}^{v} & =\left(2-\frac{\mu_{f}^{2} \tau_{\omega}}{\tau_{f}+\mu_{f}^{2} \tau_{\omega}+\tau_{p}} \frac{\mu_{a}}{\mu_{f}}\right) \delta_{x}^{v}, b_{y}^{v}=\left(2-\frac{\mu_{f}^{2} \tau_{\omega}}{\tau_{f}+\mu_{f}^{2} \tau_{\omega}+\tau_{p}} \frac{\mu_{a}}{\mu_{f}}\right) \delta_{y}^{v}, \\
b_{\omega}^{v} & =\frac{\mu_{f}^{2} \tau_{\omega}}{\tau_{f}+\mu_{f}^{2} \tau_{\omega}+\tau_{p}} \frac{1}{\mu_{f}}+\left(2-\frac{\mu_{f}^{2} \tau_{\omega}}{\tau_{f}+\mu_{f}^{2} \tau_{\omega}+\tau_{p}} \frac{\mu_{a}}{\mu_{f}}\right) \delta_{\omega}^{v} .
\end{aligned}
$$

\section{Proof of Proposition 3}

We only need to prove Part (b.iii), since all the other parts have been proved in the text. Consider the process of $\tau_{\xi} \rightarrow \infty$ or $\tau_{\xi} \rightarrow 0$. If $\lim _{\tau_{\xi}} \frac{f_{1}\left(\tau_{\xi}\right)}{f_{2}\left(\tau_{\xi}\right)}=0$, then we denote $f_{1}=o\left(f_{2}\right)$, meaning that $f_{1}$ converges at a faster rate than $f_{2}$. If $\lim _{\tau_{\xi}} \frac{f_{1}\left(\tau_{\xi}\right)}{f_{2}\left(\tau_{\xi}\right)}$ is bounded (but different from 0 ), then we denote $f_{1}=O\left(f_{2}\right)$, meaning that $f_{1}$ and $f_{2}$ converge at the same rate. By (22) and (10), we have $\phi_{y}=O(1)$ and $\tau_{p}=O\left(\tau_{\xi}\right)$. By (23) and the orders of $\phi_{y}$ and $\tau_{p}$, we have:

$$
\frac{\partial \phi_{y}}{\partial \tau_{\omega}}=-\frac{\phi_{y}}{\tau_{f}+\tau_{y}+\tau_{\omega}}+o(1)
$$


So, by (19), we have:

$$
\frac{\partial \tau_{p}}{\partial \tau_{\omega}}=-\frac{2 \phi_{y}^{2} \tau_{x} \tau_{y}^{2}}{\left(\tau_{y}+\phi_{y}^{2} \tau_{x}\right)^{2}\left(\tau_{f}+\tau_{y}+\tau_{\omega}\right)} \tau_{\xi}+o\left(\tau_{\xi}\right) .
$$

Thus, by (18), we have:

$$
\frac{\partial R E}{\partial \tau_{\omega}} \propto 1+\frac{\partial \tau_{p}}{\partial \tau_{\omega}}=1-\frac{2 \phi_{y}^{2} \tau_{x} \tau_{y}^{2}}{\left(\tau_{y}+\phi_{y}^{2} \tau_{x}\right)^{2}\left(\tau_{f}+\tau_{y}+\tau_{\omega}\right)} \tau_{\xi}+o\left(\tau_{\xi}\right) .
$$

when $\mu_{f}=1$ and $\mu_{a}=0$. As a result, $\frac{\partial R E}{\partial \tau_{\omega}}<0$ for sufficiently large $\tau_{\xi}$, and $\frac{\partial R E}{\partial \tau_{\omega}}>0$ for sufficiently small $\tau_{\xi}$.

\section{References}

Amador, M., and P. O. Weill (2010), "Learning from prices: Public Communication and Welfare," Journal of Political Economy 118, 866-907.

Angeletos, G. M.,and A. Pavan (2004), "Transparency of Information and Coordination in Economies with Investment Complementarities," American Economic Review 94, 91-98.

Angeletos, G. M., and A. Pavan (2007), "Efficient Use of Information and Social Value of Information," Econometrica 75, 1103-1142.

Bond, P., A. Edmans, and I. Goldstein (2012), "The Real Effects of Financial Markets," Annual Review of Financial Economics 4, 339-360.

Bond, P., and I. Goldstein (2012), "Government Intervention and Information Aggregation by Prices," Working Paper, University of Pennsylvania.

Chen, Q., I. Goldstein, and W. Jiang (2007), "Price Informativeness and Investment Sensitivity to Stock Price," Review of Financial Studies 20, 619-650.

Cheynel, E. (2013), "A Theory of Voluntary Disclosure and Cost of Capital," forthcoming in Review of Accounting Studies.

Dow, J., and G. Gorton (1997), "Stock Market Efficiency and Economic Efficiency: Is There a Connection?" Journal of Finance 52, 1087-1129.

Dow, J. and R. Rahi (2003), "Informed Trading, Investment, and Economic Welfare," Journal of Business 76, 430-454.

Edmans, A., I. Goldstein, and W. Jiang (2012), "The Real Effects of Financial Markets: The Impact of Prices on Takeovers," Journal of Finance 67, 933-971. 
Edmans, A., M. Heinle, and C. Huang (2013), "The Real Costs of Disclosure," Wharton Working Paper.

Foucault, T., and L. Frésard (2013), "Learning from Peers' Stock Prices and Corporate Investment," forthcoming in Journal of Financial Economics.

Froot, K., D. Scharfstein, and J. Stein (1992), "Herd on the Street: Informational Inefficiencies in a Market with Short-Term Speculation," Journal of Finance 47, 1461-1484.

Gao, P. (2010). "Disclosure Quality, Cost of Capital, and Investor Welfare," Accounting Review 85, 1-29.

Gao, P., and P. J. Liang (2013), "Informational Feedback, Adverse Selection, and Optimal Disclosure Policy," Journal of Accounting Research 51, 1133-1158.

Goldman, E. (2005), "Organizational Form, Information Collection, and the Value of the Firm," Journal of Business 78, 817-839.

Goldstein, I., E. Ozdenoren, and K. Yuan (2011), "Learning and Complementarities in Speculative Attacks," Review of Economic Studies 78, 263-292.

Goldstein, I., E. Ozdenoren, and K. Yuan (2013), "Trading Frenzies and Their Impact on Real Investment," forthcoming in Journal of Financial Economics.

Goldstein, I., and L. Yang (2013), "Market Efficiency and Real Efficiency: The Connect and Disconnect Via Feedback Effects," Working Paper.

Greenstone, M., P. Oyer, and A. Vissing-Jorgensen (2006), "Mandated Disclosure, Stock Returns, and the 1964 Securities Acts Amendments," Quarterly Journal of Economics 121, 399-460.

Greenwood, J., G. M. MacDonald, and G-J. Zhang (1996), "The Cyclical Behavior of Job Creation and Job Destruction: A Sectoral Model," Economic Theory 7, 95-112.

Hakannson, N. H., J. Kunkel, and J. Ohlson (1982), "Sufficient and Necessary Conditions for Information to Have Social Value in Pure Exchange," Journal of Finance 37, 11691181.

Han, B., Y.-J. Liu, Y. Tang, L. Yang, and L. Yu (2013), "Disclosure and Efficiency in Noise-Driven Markets," Working Paper.

Hirshleifer, J. (1971), "The Private and Social Value of Information and the Reward to Inventive Activity," American Economic Review 61, 561-574. 
Kanodia, C. Accounting Disclosure and Real Effects. Hanover, MA: Now Publishers Inc., 2007.

Kondor, P. (2012), "The More We Know about the Fundamental, the Less We Agree on the Price," Review of Economic Studies 79), 1175-1207.

Kyle, A. S. (1985), "Continuous Auctions and Insider Trading," Econometrica 53, 13151335.

Liu, Z., P. Wang, and T. Zha (2013), "Land-Price Dynamics and Macroeconomic Fluctuations," Econometrica 81, 1147-1184.

Luo, Y. (2005), "Do Insiders Learn from Outsiders? Evidence From Mergers and Acquisitions," Journal of Finance 60, 1951-1982.

Morris, S., and H. S. Shin (2002), "The Social Value of Public Information," American Economic Review 92, 1521-1534.

Veldkamp, L., and J. Wolfers (2007), "Aggregate Shocks or Aggregate Information? Costly Information and Business Cycle Comovement," Journal of Monetary Economics 54(S), $37-55$. 
Figure 1: The Effect of Disclosure about Factor $\widetilde{A}$

(a)

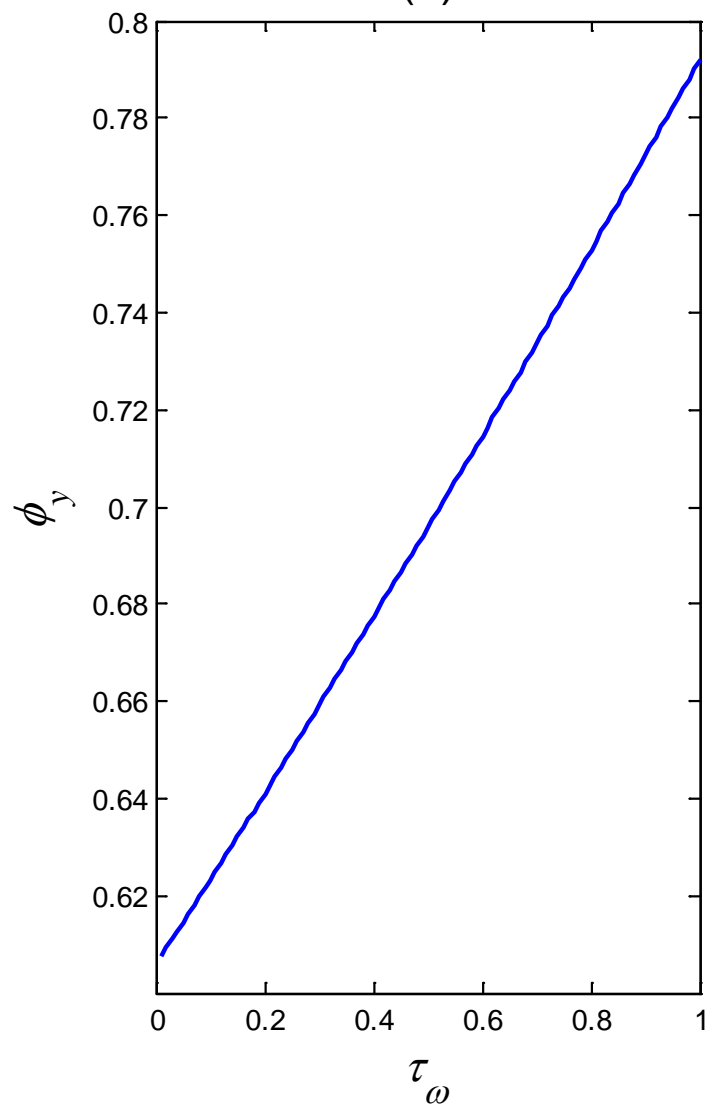

(b)

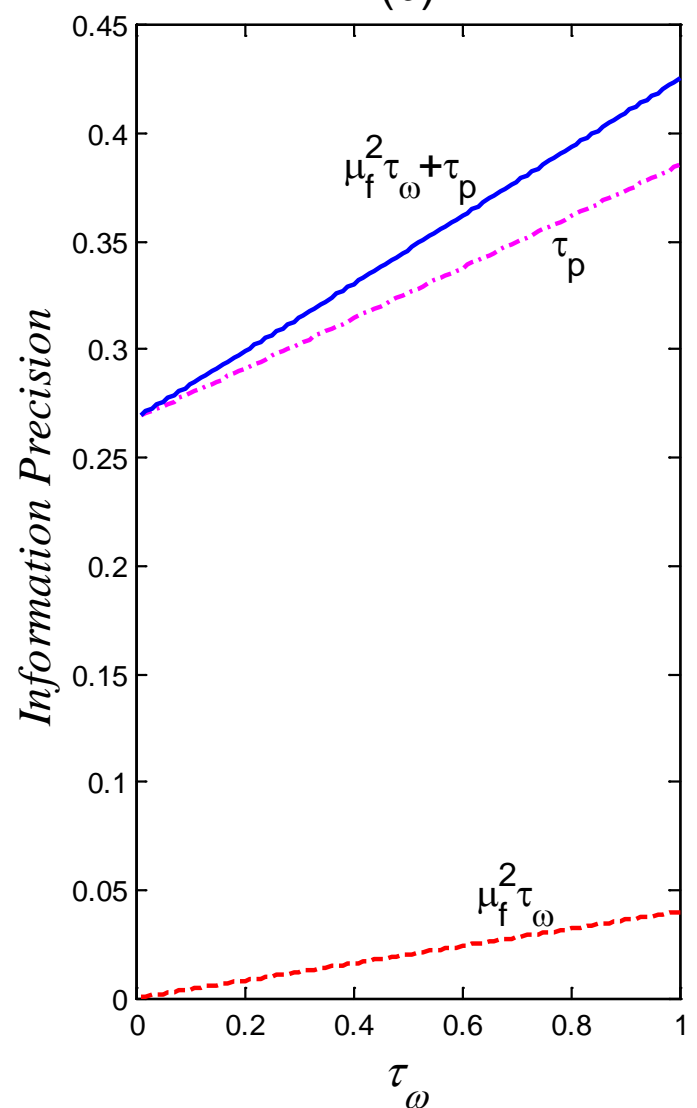

This figure plots the trading and efficiency implications of public information release when the public signal is primarily a signal about factor $\tilde{a}$. Parameter $\tau_{\omega}$ controls the precision of public information. The other parameter values are: $\mu_{a}=0.8, \mu_{f}=0.2, \tau_{a}=\tau_{f}=\tau_{x}=\tau_{y}=\tau_{\xi}=1$, and $\lambda=2$. 


\section{Figure 2: The Effect of Disclosure about Factor $\widetilde{F}$ in Economies Aggregating Private Information Effectively}

(a)

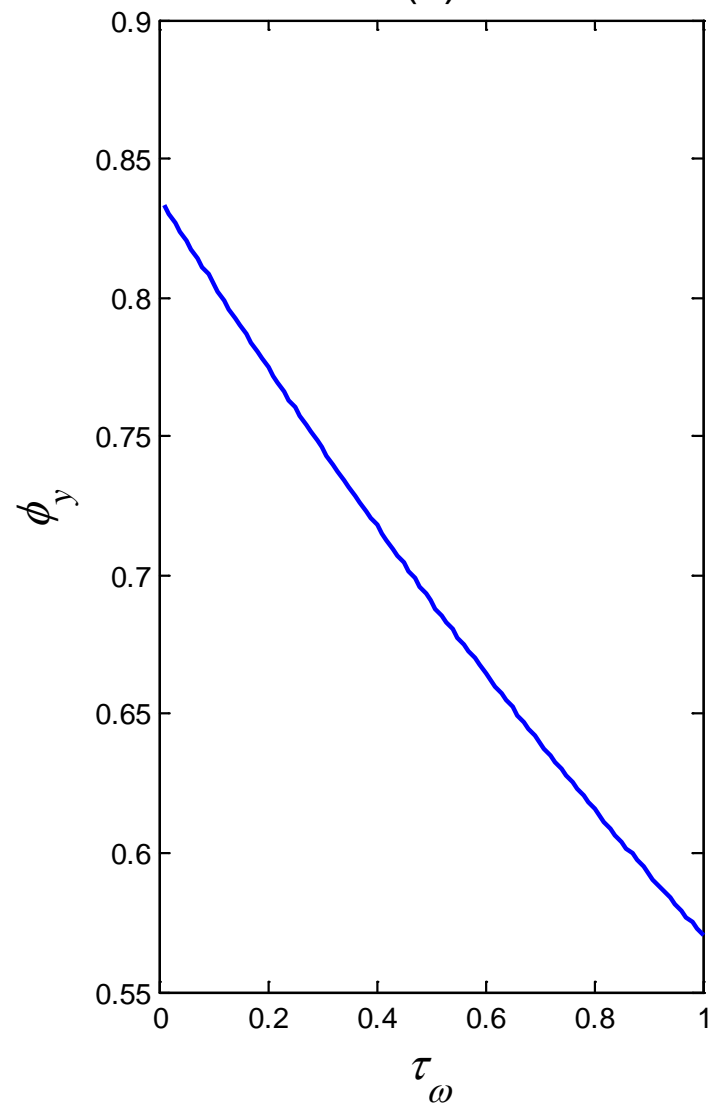

(b)

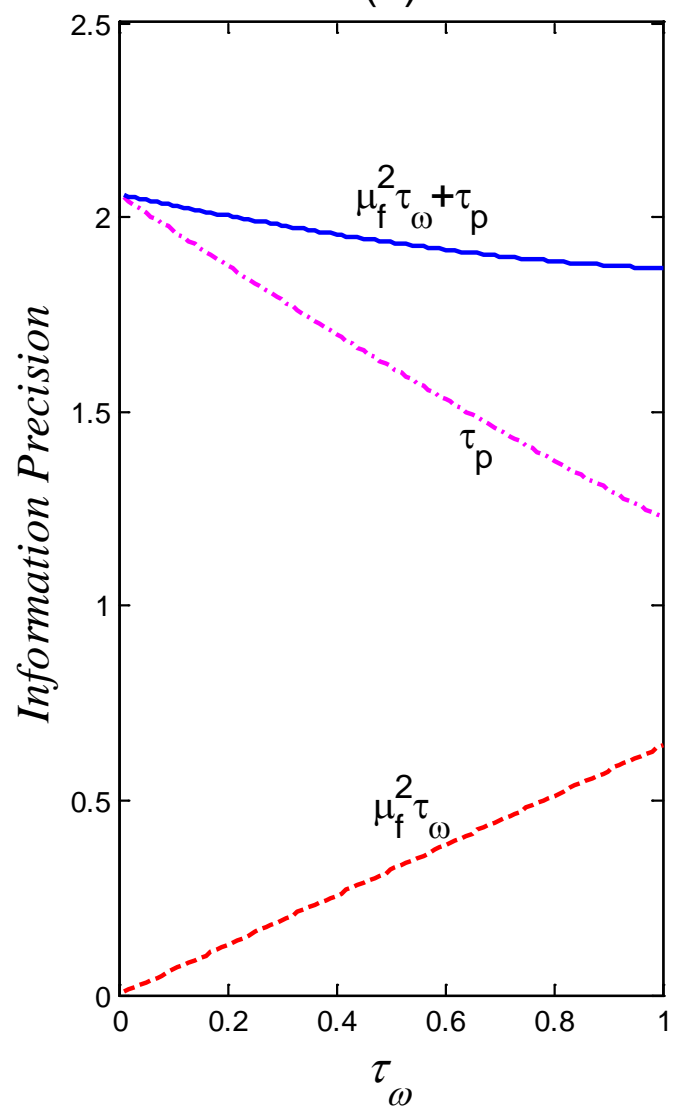

This figure plots the trading and efficiency implications of public information release when the public signal is primarily a signal about factor $\tilde{f}$ and when the market aggregates private information effectively. Parameter $\tau_{\omega}$ controls the precision of public information. The other parameter values are: $\mu_{a}=0.2, \mu_{f}=0.8, \tau_{a}=\tau_{f}=\tau_{x}=\tau_{y}=1, \tau_{\xi}=5$, and $\lambda=2$. 


\section{Figure 3: The Effect of Disclosure about Factor $\widetilde{F}$ in Economies Aggregating Private Information Ineffectively}

(a)

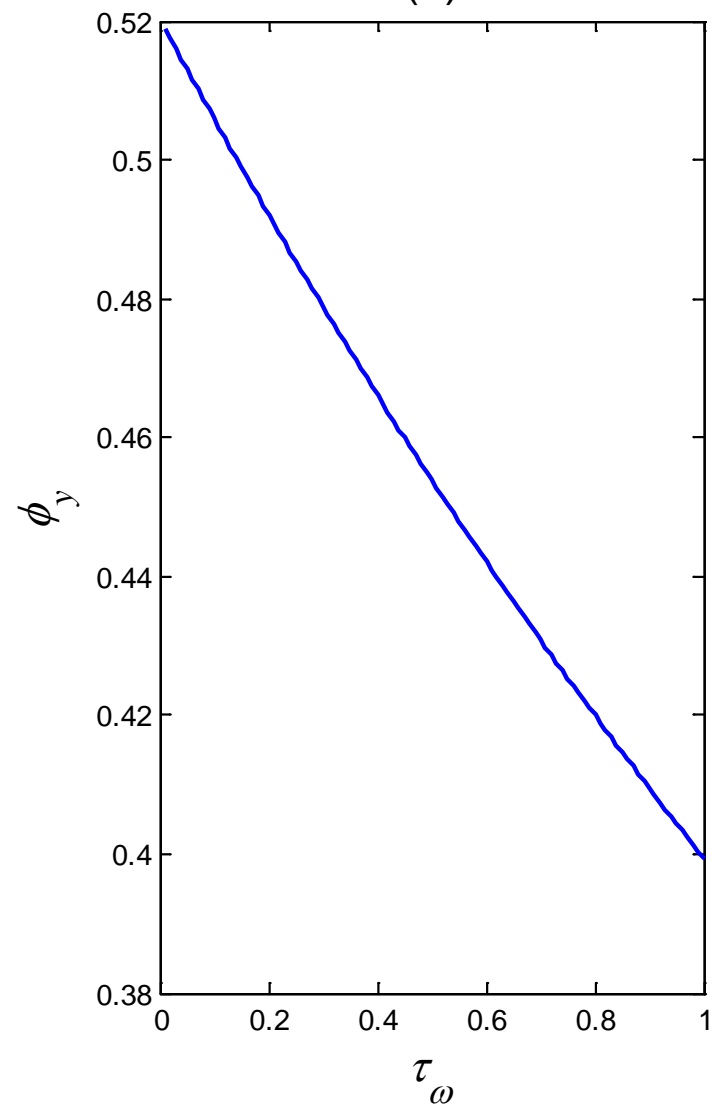

(b)

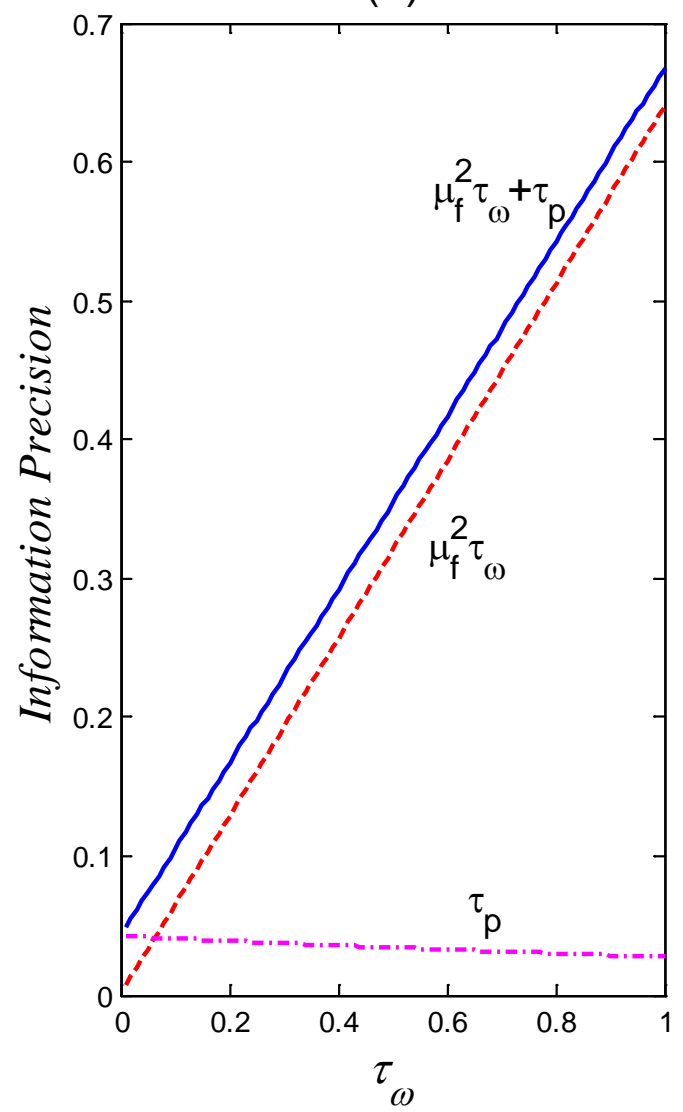

This figure plots the trading and efficiency implications of public information release when the public signal is primarily a signal about factor $\tilde{f}$ and when the market aggregates private information ineffectively. Parameter $\tau_{\omega}$ controls the precision of public information. The other parameter values are: $\mu_{a}=0.2, \mu_{f}=0.8, \tau_{a}=\tau_{f}=\tau_{x}=\tau_{y}=1, \tau_{\xi}=0.2$, and $\lambda=2$. 


\section{Figure 4: The Effect of Public Disclosure in Extended Economies}

(a1)

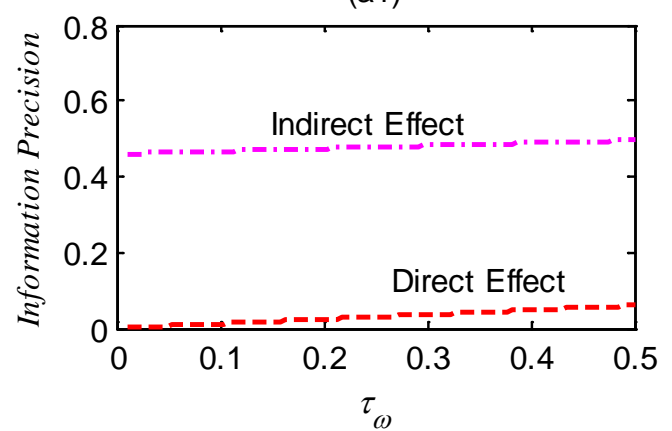

(b1)

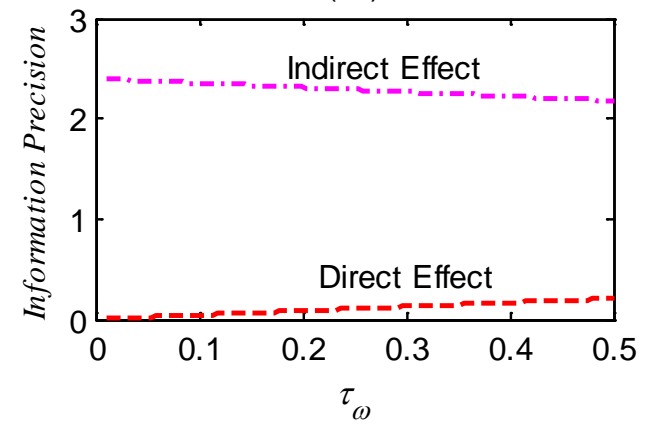

(c1)

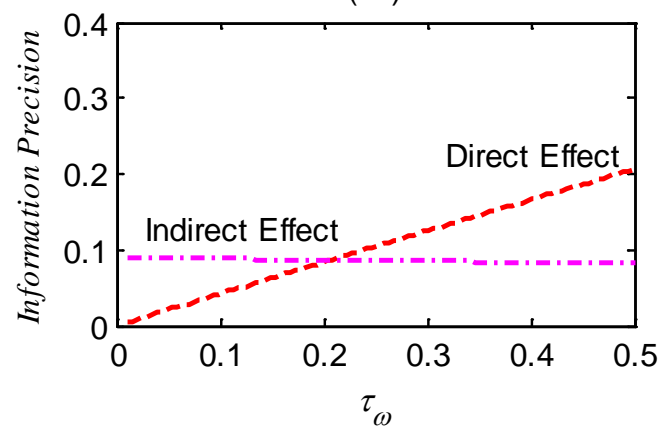

(a2)

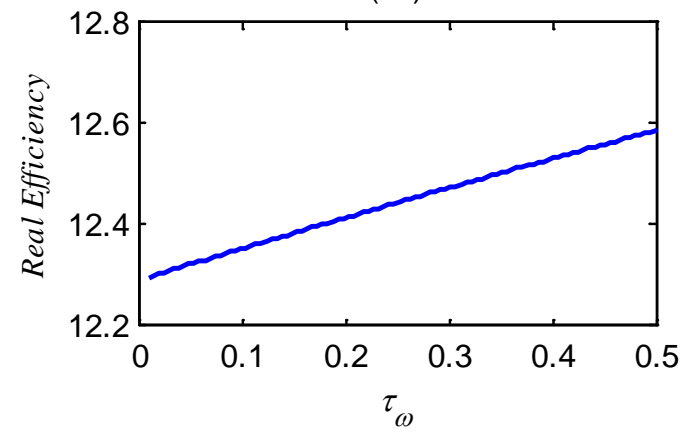

(b2)

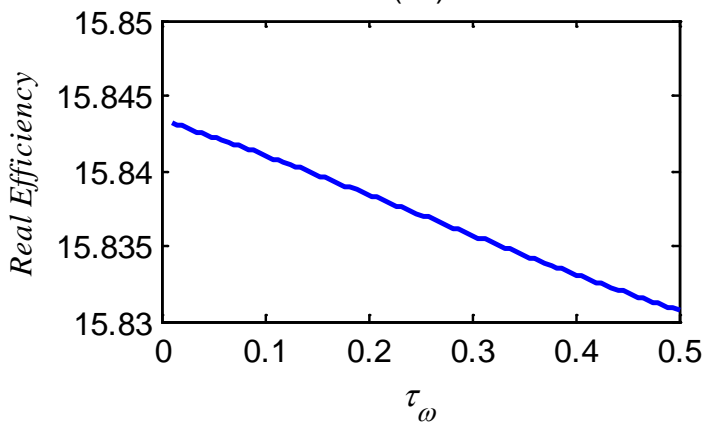

(c2)

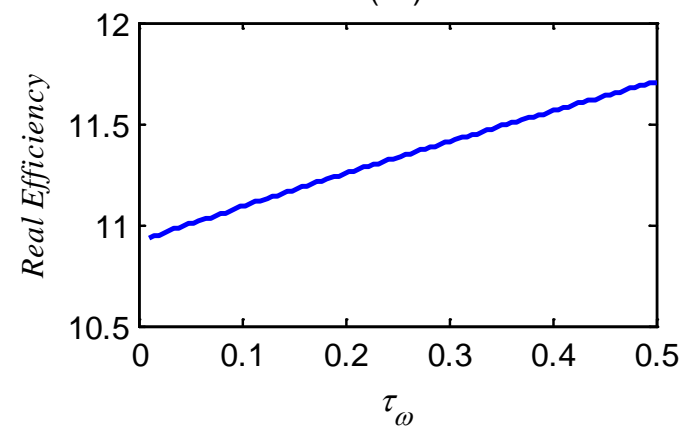

This figure plots the efficiency implications of public information in the extended economies in which capital provider receive noisy signals about factors $\tilde{a}$ and $\tilde{f}$. The left panels plot the direct and indirect effects of disclosure on capital providers forecast. The direct effect refers to the precision acquired by capital providers from observing the public information. The indirect effect refers to the precision acquired from the price whose information amount is affected by public information. The right panels plot real efficiency against the precision of public disclosure. In all panels, we have set $\tau_{a}=\tau_{f}=\tau_{y}=\tau_{s}=\tau_{\xi}=1, \tau_{z}=5, \beta=1 / 2, c_{0}=1$, and $\lambda=2$. In Panels (a1)-(a2), $\mu_{a}=0.8, \mu_{f}=0.2$, and $\tau_{\xi}=1$. In Panels (b1)-(b2), $\mu_{a}=0.2, \mu_{f}=0.8$, and $\tau_{\xi}=5$. In Panels (c1)-(c2), $\mu_{a}=0.2, \mu_{f}=0.8$, and $\tau_{\xi}=0.2$. 